\title{
Biflavanones, Chalconoids, and Flavonoid Analogues from the Stem Bark of Ochna holstii
}

Thobias M. Kalenga, Monica M. Ndoile, Yoseph Atilaw, Pieter J. Gilissen, Joan J. E. Munissi, Anastasia Rudenko, Catarina Bourgard, Per Sunnerhagen, Stephen S. Nyandoro,* and Mate Erdelyi*

Cite This: J. Nat. Prod. 2021, 84, 364-372

Read Online

ACCESS

Llll Metrics \& More

Article Recommendations

Supporting Information

ABSTRACT: Two new biflavanones (1 and 2), three new bichalconoids (3-5), and 11 known flavonoid analogues (6-16) were isolated from the stem bark extract $\left(\mathrm{CH}_{3} \mathrm{OH}-\mathrm{CH}_{2} \mathrm{Cl}_{2}, 7: 3\right.$, $\mathrm{v} / \mathrm{v}$ ) of Ochna holstii. The structures of the isolated metabolites were elucidated by NMR spectroscopic and mass spectrometric analyses. The crude extract and the isolated metabolites were evaluated for antibacterial activity against Bacillus subtilis (Grampositive) and Escherichia coli (Gram-negative) as well as for cytotoxicity against the MCF-7 human breast cancer cell line. The crude extract and holstiinone A (1) exhibited moderate antibacterial activity against $B$. subtilis with $\mathrm{MIC}$ values of 9.1 $\mu \mathrm{g} / \mathrm{mL}$ and $14 \mu \mathrm{M}$, respectively. The crude extract and lophirone $\mathrm{F}$ (14) showed cytotoxicity against MCF-7 with $\mathrm{EC}_{50}$ values of 11
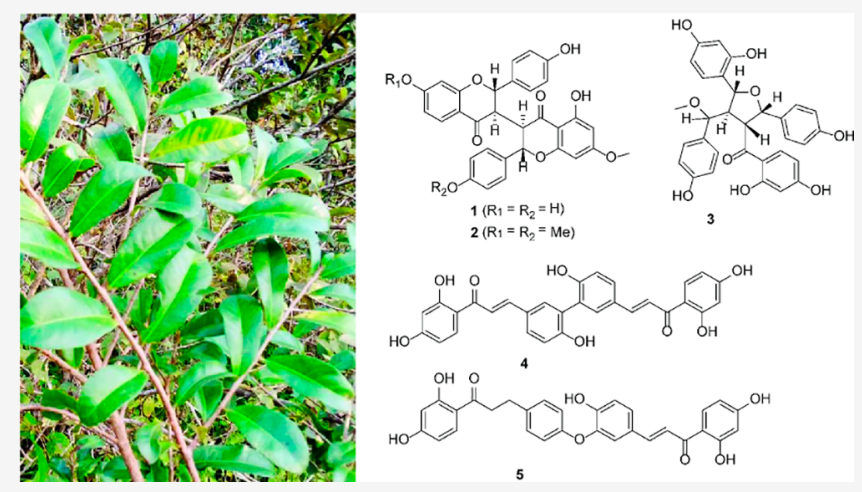
$\mu \mathrm{g} / \mathrm{mL}$ and $24 \mu \mathrm{M}$, respectively. The other isolated metabolites showed no significant antibacterial activities $(\mathrm{MIC}>250 \mu \mathrm{M})$ and cytotoxicities $\left(\mathrm{EC}_{50} \geq 350 \mu \mathrm{M}\right)$.

$\mathrm{T}$ he genus Ochna (Ochnaceae) comprises about 86 species of evergreen trees or shrubs distributed in Madagascar and in tropical Africa, Asia, and the America. ${ }^{1}$ Members of this genus are used in traditional medicine to treat malaria, diarrhea, and hemorrhoids, as well as microbial and helminthic ailments. ${ }^{1,2}$ Many species from the genus Ochna are known to metabolize biflavanones, chalcones, and related compounds ${ }^{3,4}$ which are reported to exhibit antimalarial and antibacterial activity and cytotoxicity against cancer cells. ${ }^{3-8}$ In our endeavor to search for bioactive natural products from Tanzanian medicinal plants, we investigated extracts of $O$. holstii. It is a shrub or a medium-sized tree up to $15 \mathrm{~m}$ high occurring in Southern Asia, Republic of South Africa, Ethiopia, Zimbabwe, Kenya, and Tanzania. ${ }^{9-11}$ The plant is locally known as "mkumbi" in Tanzania. There is no ethnomedical information reported for this plant species, apart from its use to relinquish evil spirits from children. The crude extract of $O$. holstii has been reported to exhibit anti-HIV and contraceptive activities. $^{12,13}$ Previous phytochemical investigations of $O$. holstii revealed the presence of dimeric and monomeric flavonoids. ${ }^{14}$ Herein, we evaluated the crude extract of the stem bark of $O$. holstii and its isolated constituents $(\mathbf{1}-\mathbf{1 6})$ for antibacterial activity against Bacillus subtilis and Escherichia coli and for cytotoxicity against the MCF-7 human breast cancer cell line.

\section{RESULTS AND DISCUSSION}

Systematic purification of the $\mathrm{CH}_{3} \mathrm{OH}-\mathrm{CH}_{2} \mathrm{Cl}_{2}(7: 3, \mathrm{v} / \mathrm{v})$ extract of the stem bark of $O$. holstii using silica gel 60 (230400 mesh) column chromatography, followed by gel filtration on a Sephadex LH-20 column and preparative reverse-phase HPLC, yielded five new metabolites (1-5) and 11 known compounds (6-16) (Figures S1-S84, Supporting Information). The structures of the isolated compounds were elucidated by $1 \mathrm{D}$ and $2 \mathrm{D}$ NMR spectroscopic and mass spectrometric analyses. The known compounds were identified as ouratein $\mathrm{D}(6),{ }^{15}$ isochamaejasmin $\mathrm{A}(7),{ }^{16,17} 7^{\prime}, 7^{\prime \prime}$-di- $O$ methylisochamaejasmin (8), ${ }^{18}$ campylospermone A (9), ${ }^{19}$ liquiritigeninyl-(1-3,II-3)-naringenin $(\mathbf{1 0}),{ }^{8}$ isoliquitigenin (11), ${ }^{20}$ terminalionone (12), ${ }^{21}$ flavumchalcone (13), ${ }^{22}$ lophirone F (14), ${ }^{23}$ 2,3-dihydrocalodenin B (15), ${ }^{3}$ and calodenin B (16) ${ }^{24,25}$ by comparison of their spectroscopic data with reported data.

Compound 1 was isolated as a pale white solid and was assigned the molecular formula $\mathrm{C}_{31} \mathrm{H}_{24} \mathrm{O}_{9}$ based on HRESIMS $\left([\mathrm{M}+\mathrm{H}]^{+} \mathrm{m} / \mathrm{z}\right.$ 541.1487, calcd 541.1499, Figure S8,

Received: September 18, 2020

Published: January 29, 2021 

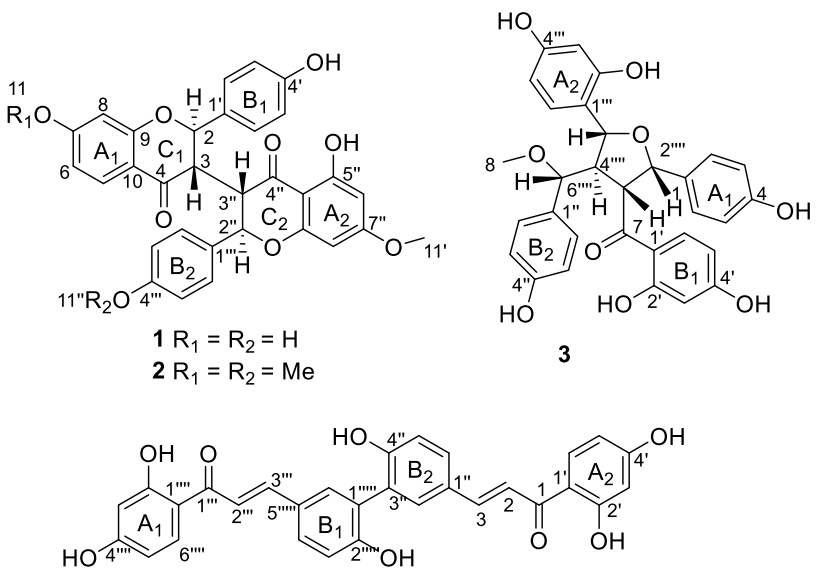

4

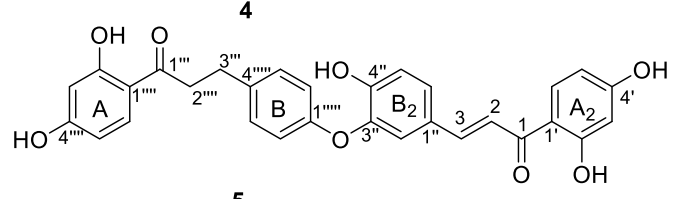

Supporting Information) and NMR data (Table 1, Figures S1S7, Supporting Information). It showed a specific rotation $[\alpha]_{D}^{24}$ of $-115\left(c 0.013, \mathrm{CH}_{3} \mathrm{OH}\right)$. The IR spectrum revealed the presence of hydroxy $\left(3396 \mathrm{~cm}^{-1}\right)$ and aromatic $\mathrm{C}=\mathrm{C}$ (1604, 1516, and $1453 \mathrm{~cm}^{-1}$ ) groups. UV absorption maxima at 262 and $330 \mathrm{~nm}$ indicated the presence of a flavanone-type structure. $^{15,26}$

The ${ }^{1} \mathrm{H}$ NMR spectrum (Table 1, Figure S1, Supporting Information) of $\mathbf{1}$ suggested that the compound has four distinct aromatic systems. Ring $A_{1}$ contained an $A B X$ spin system resonating at $\delta_{\mathrm{H}} 7.50(\mathrm{H}-5), 6.54(\mathrm{H}-6)$, and $6.45(\mathrm{H}-$ $8)$. The substitution pattern of ring $\mathrm{A}_{2}$ was deduced from the coupling constant $\left({ }^{4} J_{6^{\prime \prime}, 8^{\prime \prime}}=2.4 \mathrm{~Hz}\right)$ of the protons at $\delta_{\mathrm{H}} 6.12$ $\left(\mathrm{H}-6^{\prime \prime}\right)$ and $6.16\left(\mathrm{H}-8^{\prime \prime}\right)$. Furthermore, compound 1 contained two protons of a $p$-disubstituted aromatic system resonating at $\delta_{\mathrm{H}} 7.28\left(\mathrm{H}-2^{\prime} / 6^{\prime}\right)$ and $6.76\left(\mathrm{H}-3^{\prime} / 5^{\prime}\right)$ for ring $\mathrm{B}_{1}$ and at $\delta_{\mathrm{H}}$ $7.25\left(\mathrm{H}-2^{\prime \prime \prime} / 6^{\prime \prime \prime}\right)$ and $6.74\left(\mathrm{H}-3^{\prime \prime \prime} / 5^{\prime \prime \prime}\right)$ for ring $\mathrm{B}_{2}$. In addition, the ${ }^{1} \mathrm{H}$ NMR spectrum displayed methoxy group protons at $\delta_{\mathrm{H}}$ $3.77\left(\mathrm{H}_{3}-11\right)$, two oxymethine protons at $\delta_{\mathrm{H}} 5.62(\mathrm{H}-2)$ and $5.58\left(\mathrm{H}-2^{\prime \prime}\right)$, and two methine protons at $\delta_{\mathrm{H}} 4.50(\mathrm{H}-3)$ and $4.46\left(\mathrm{H}-3^{\prime \prime}\right)$. The 2,3-relative configuration of compound 1 was determined via the ${ }^{1} \mathrm{H}$ NMR coupling constants and splitting patterns. The splitting patterns of the resonances assigned to $\mathrm{H}-2(\mathrm{~d}, J=10.2 \mathrm{~Hz}), \mathrm{H}-2^{\prime \prime}(\mathrm{d}, J=10.0 \mathrm{~Hz}), \mathrm{H}-3$ $(\mathrm{dd}, J=10.2,7.8 \mathrm{~Hz})$ and $\mathrm{H}-3^{\prime \prime}(\mathrm{dd}, J=10.0,7.8 \mathrm{~Hz})$ suggested rings $\mathrm{C}_{1}(\mathrm{H}-2 / \mathrm{H}-3)$ and $\mathrm{C}_{2}\left(\mathrm{H}-2^{\prime \prime} / \mathrm{H}-3^{\prime \prime}\right)$ to have trans-relative configurations. This conclusion is corroborated by the observed ${ }^{3} J_{2,3}$ being larger than that $(2.3 \mathrm{~Hz})$ reported by Rocha et al. for the 2,3-cis-configured analogues, but similar to the 9.2 and $9.9 \mathrm{~Hz}$ reported for the trans relative configured analogues. ${ }^{15}$ Protons $\mathrm{H}-3$ and $\mathrm{H}-3^{\prime \prime}$ showed ${ }^{3} J_{3,3^{\prime \prime}}=7.8 \mathrm{~Hz}$, which is comparable to the ${ }^{3} J_{3,3 "}=8.5 \mathrm{~Hz}$ previously reported

Table 1. NMR Spectroscopic Data (500 MHz) for Holstiinone A (1) and Holstiinone B (2)

\begin{tabular}{|c|c|c|c|c|c|c|}
\hline \multirow[b]{2}{*}{ position } & \multicolumn{3}{|c|}{ holstiinone A (methanol- $d_{4}$ ) } & \multicolumn{3}{|c|}{ holstiinone $\mathrm{B}\left(\mathrm{CDCl}_{3}\right)$} \\
\hline & $\delta_{\mathrm{C}}$, type & $\delta_{\mathrm{H}}(J$ in $\mathrm{Hz})$ & $\mathrm{HMBC}, \mathrm{H} \rightarrow \mathrm{C}$ & $\delta_{\mathrm{C}}$, type & $\delta_{\mathrm{H}}(J$ in $\mathrm{Hz})$ & $\mathrm{HMBC}, \mathrm{H} \rightarrow \mathrm{C}$ \\
\hline 2 & 86.7, $\mathrm{CH}$ & $5.62, \mathrm{~d}(10.2)$ & $\mathrm{C}-2^{\prime} / 6^{\prime}, \mathrm{C}-3^{\prime \prime}, \mathrm{C}-4, \mathrm{C}-9$ & $85.3, \mathrm{CH}$ & $5.66, \mathrm{~d}(10.1)$ & $\mathrm{C}-3, \mathrm{C}-3^{\prime}, \mathrm{C}-2^{\prime \prime} / 6^{\prime \prime}, 4,4^{\prime}$ \\
\hline 3 & $60.4, \mathrm{CH}$ & 4.50, dd $(10.2,7.8)$ & $\mathrm{C}-2^{\prime}, \mathrm{C}-4, \mathrm{C}-4^{\prime}, \mathrm{C}-10$ & $59.8, \mathrm{CH}$ & 4.49, dd $(10.1,8.2)$ & C-3", C-1", C-2, C-4, C-4" \\
\hline 4 & 196.0, C & & & 194.2, C & & \\
\hline 5 & $131.9, \mathrm{CH}$ & $7.50, \mathrm{~d}(8.2)$ & C-4, C-7, C-9 & 131.1, $\mathrm{CH}$ & $7.66, \mathrm{~d}(8.8)$ & C-4, C-7, C-9 \\
\hline 6 & $112.8, \mathrm{CH}$ & $6.54, \mathrm{dd}(8.2,2.4)$ & C-7, C-8, C-10 & $111.0, \mathrm{CH}$ & $6.62, \mathrm{dd}(8.8,2.4)$ & $\mathrm{C}-7, \mathrm{C}-8, \mathrm{C}-10$ \\
\hline 7 & $165.3, \mathrm{C}$ & & & $164.9, \mathrm{C}$ & & \\
\hline 8 & $107.9, \mathrm{CH}$ & $6.45, \mathrm{~d}(2.4)$ & C-7, C-9, C-10, C-6 & 105.1, CH & $6.55, \mathrm{~d}(2.4)$ & $\mathrm{C}-6, \mathrm{C}-7, \mathrm{C}-10$ \\
\hline 9 & $166.5, \mathrm{C}$ & & & 165.7, C & & \\
\hline 10 & 122.1, C & & & $121.9, \mathrm{C}$ & & \\
\hline $1^{\prime}$ & 132.1, C & & & 132.0, C & & \\
\hline $2^{\prime} / 6^{\prime}$ & 129.3, CH & $7.25, \mathrm{AA}^{\prime}$ & $\mathrm{C}-2^{\prime \prime}, \mathrm{C}-3^{\prime} / 5^{\prime}, \mathrm{C}-4^{\prime}$ & 128.2, $\mathrm{CH}$ & $7.34, \mathrm{AA}^{\prime}$ & $\mathrm{C}-2, \mathrm{C}-3^{\prime} / 5^{\prime}, \mathrm{C}-4^{\prime}$ \\
\hline $3^{\prime} / 5^{\prime}$ & 116.3, $\mathrm{CH}$ & $6.76, \mathrm{XX}^{\prime}$ & $\mathrm{C}-2^{\prime} / 6^{\prime}, \mathrm{C}-1^{\prime}, \mathrm{C}-4^{\prime}$ & 114.1, $\mathrm{CH}$ & $6.85, \mathrm{XX}^{\prime}$ & $\mathrm{C}-2^{\prime} / 6^{\prime}, \mathrm{C}-4^{\prime}, \mathrm{C}-1^{\prime}$ \\
\hline $4^{\prime}$ & $158.7, \mathrm{C}$ & & & $159.7, \mathrm{C}$ & & \\
\hline $2^{\prime \prime}$ & $86.4, \mathrm{CH}$ & $5.58, \mathrm{~d}(10.0)$ & $\mathrm{C}-2^{\prime \prime \prime} / 6^{\prime \prime \prime}, \mathrm{C}-3^{\prime \prime}, \mathrm{C}-4^{\prime \prime}, \mathrm{C}-9^{\prime \prime}$ & $85.2, \mathrm{CH}$ & $5.74, \mathrm{~d}(10.1)$ & $\mathrm{C}-2^{\prime \prime \prime} / 6^{\prime \prime \prime}, \mathrm{C}-9^{\prime \prime}, \mathrm{C}-4^{\prime \prime}, \mathrm{C}-3^{\prime \prime}$ \\
\hline $3^{\prime \prime}$ & $60.2, \mathrm{CH}$ & $4.46, \mathrm{dd}(10.0,7.8)$ & C-2, C-2", C-4, C- $4^{\prime \prime}, C-1^{\prime \prime \prime}$ & $59.5, \mathrm{CH}$ & $4.45, \mathrm{dd}(10.1,8.2)$ & $\mathrm{C}-2, \mathrm{C}-3, \mathrm{C}-4, \mathrm{C}-4^{\prime \prime}, \mathrm{C}-1^{\prime \prime \prime}$ \\
\hline $4^{\prime \prime}$ & $200.5, \mathrm{C}$ & & & 198.6, C & & \\
\hline $5^{\prime \prime}$ & $165.3, \mathrm{C}$ & & & 164.6, C & & \\
\hline $6^{\prime \prime}$ & 97.7, $\mathrm{CH}$ & $6.12, \mathrm{~d}(2.4)$ & $\mathrm{C}-7^{\prime \prime}, \mathrm{C}-8^{\prime \prime}, \mathrm{C}-10^{\prime \prime}$ & $96.9, \mathrm{CH}$ & $6.14, \mathrm{~d}(2.4)$ & $C-5^{\prime \prime}, C-10^{\prime \prime}, C-8^{\prime \prime}, C-7^{\prime \prime}$ \\
\hline $7^{\prime \prime}$ & $167.9, \mathrm{C}$ & & & $166.4, \mathrm{C}$ & & \\
\hline $8^{\prime \prime}$ & 99.9, $\mathrm{CH}$ & $6.16, \mathrm{~d}(2.4)$ & $\mathrm{C}-6^{\prime \prime}, \mathrm{C}-7^{\prime \prime}, \mathrm{C}-10^{\prime \prime}$ & $99.1, \mathrm{CH}$ & $6.11, \mathrm{~d}(2.4)$ & $\mathrm{C}-10^{\prime \prime}, \mathrm{C}-6^{\prime \prime}, \mathrm{C}-7^{\prime \prime}$ \\
\hline $9^{\prime \prime}$ & $166.9, \mathrm{C}$ & & & $165.1, \mathrm{C}$ & & \\
\hline $10^{\prime \prime}$ & 109.7, C & & & $108.8, \mathrm{C}$ & & \\
\hline $1^{\prime \prime \prime}$ & 131.8, C & & & 132.5, C & & \\
\hline $2^{\prime \prime \prime} / 6^{\prime \prime \prime}$ & 129.5, CH & $7.28, \mathrm{AA}^{\prime}$ & $\mathrm{C}-2,3^{\prime} / 5^{\prime}, \mathrm{C}-4^{\prime}$ & $128.7, \mathrm{CH}$ & 7.36, $\mathrm{AA}^{\prime}$ & $C-2^{\prime \prime}, C-4^{\prime \prime \prime}, C-3^{\prime \prime \prime} / 5^{\prime \prime \prime}$ \\
\hline $3^{\prime \prime \prime} / 5^{\prime \prime \prime}$ & 116.2, $\mathrm{CH}$ & $6.74, \mathrm{XX}^{\prime}$ & $\mathrm{C}-2^{\prime \prime \prime} / 6^{\prime \prime \prime}, \mathrm{C}-4^{\prime \prime \prime}, \mathrm{C}-1^{\prime \prime \prime}$ & $115.5, \mathrm{CH}$ & $7.79, \mathrm{XX}^{\prime}$ & $\mathrm{C}-2^{\prime \prime \prime} / 6^{\prime \prime \prime}, \mathrm{C}-1^{\prime \prime \prime}, \mathrm{C}-4^{\prime \prime \prime}$ \\
\hline $4^{\prime \prime \prime}$ & $158.7, \mathrm{C}$ & & & $155.7, \mathrm{C}$ & & \\
\hline $\mathrm{OCH}_{3}-11$ & $56.2, \mathrm{CH}$ & $3.77, \mathrm{~s}$ & $\mathrm{C}-7^{\prime \prime}$ & $55.8, \mathrm{CH}_{3}$ & $3.76, \mathrm{~s}$ & $\mathrm{C}-7$ \\
\hline $\mathrm{OCH}_{3}-11^{\prime \prime}$ & & & & $55.4, \mathrm{CH}_{3}$ & $3.79, \mathrm{~s}$ & $\mathrm{C}-7^{\prime \prime}$ \\
\hline $\mathrm{OCH}_{3}-7^{\prime \prime \prime}$ & & & & $55.8, \mathrm{CH}_{3}$ & $3.78, \mathrm{~s}$ & $\mathrm{C}-4^{\prime \prime \prime}$ \\
\hline $\mathrm{OH}-5^{\prime}$ & & & & & $11.9, \mathrm{~s}$ & $C-5^{\prime}, C-6^{\prime}, C-10^{\prime}$ \\
\hline
\end{tabular}


for the gauche conformation, but dissimilar to the ${ }^{3} J_{3,3^{\prime \prime}}=12.0$ $\mathrm{Hz}$ of the anti-oriented protons of structurally closely related compounds. ${ }^{6}$ Based on this observation, $\mathbf{1}$ is proposed to possess syn $\mathrm{H}-3 / \mathrm{H}-3$ " geometry. The HMBC cross-peaks (Table 1, Figure S5, Supporting Information) of $\mathrm{H}-11\left(\delta_{\mathrm{H}}\right.$ 3.77), H-6" $\left(\delta_{\mathrm{H}} 6.12\right)$, and $\mathrm{H}-8^{\prime \prime}\left(\delta_{\mathrm{H}} 6.16\right)$ to C-7" $\left(\delta_{\mathrm{C}} 167.9\right)$ corroborated the location of the methoxy group at C-7" of ring $\mathrm{A}_{2}$. In addition, the HMBC cross-peaks of proton resonances at $\delta_{\mathrm{H}} 4.50(\mathrm{H}-3)$ to C-2" $\left(\delta_{\mathrm{C}} 86.4\right), \mathrm{C}-4\left(\delta_{\mathrm{C}} 196.0\right), \mathrm{C}-4^{\prime \prime}\left(\delta_{\mathrm{C}}\right.$ $200.5)$, and C-10 $\left(\delta_{\mathrm{C}} 122.1\right)$ and those at $\delta_{\mathrm{H}} 5.62(\mathrm{H}-2)$ to C$3^{\prime \prime}\left(\delta_{\mathrm{C}} 60.2\right), \mathrm{C}-4\left(\delta_{\mathrm{C}} 196.0\right), \mathrm{C}-9\left(\delta_{\mathrm{C}} 166.4\right)$, and C-2'/6' $\left(\delta_{\mathrm{C}}\right.$ $129.5)$ established the $\mathrm{C}-3-\mathrm{C}-3^{\prime \prime}$ linkage of the two flavanone units. Similar C-3-C-3" linkages were previously reported from other plant species including campylospermone B from Campylospermum mannii, ${ }^{19}$ ouratein D from Ouratea spectabilis, ${ }^{15}$ 4,4,7-tri-O-methylisocapylospermone A from Ochna serrulata, $^{27}$ chamaejasmin from Ormocarpum kirkii, ${ }^{8}$ and chamaejasmin D from Stellera chamaejasme. ${ }^{28}$ The NMR spectroscopic data for compound 1 closely resembled those of campylospermone $\mathrm{B}^{19}$ and liquiritigeninyl-(1-3,II-3)-naringenin, ${ }^{8}$ but had a methoxy group resonating at $\delta_{\mathrm{H}} 3.77(\mathrm{H}-11)$ and attached to C-7" $\left(\delta_{\mathrm{C}} 167.9\right)$, indicated by the HMBC cross-peak of H-11 $\left(\delta_{\mathrm{H}} 3.77\right)$ with C-7" $\left(\delta_{\mathrm{C}} 167.9\right)$. The ECD spectrum of 1 (Figure 1) showed a weak positive Cotton effect

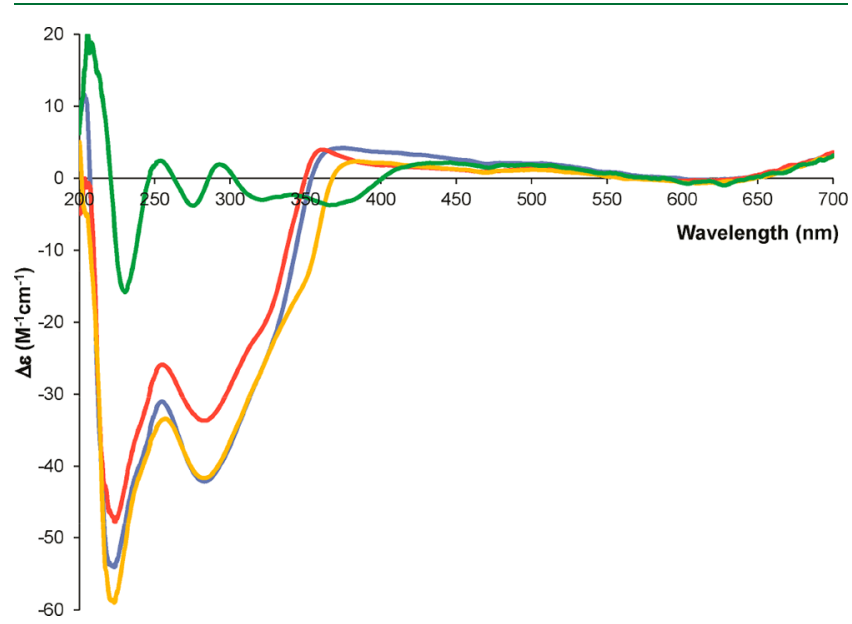

Figure 1. ECD spectra of holstiinone A (1, blue), holstiinone B (2, red), holstiichalcone I (3, yellow), and holstiichalcone II (4, green) in $\mathrm{MeOH}$.

for the $\pi \rightarrow \pi^{*}$ transition at ca. $360 \mathrm{~nm}$, a negative Cotton effect for the $\mathrm{n} \rightarrow \pi^{*}$ transition at ca. $300 \mathrm{~nm}$, and a negative Cotton effect at ca. $210 \mathrm{~nm}$ for the ${ }^{1} \mathrm{La}$ electronic transition, which indicated a $(2 R)$ absolute configuration for each flavanone moiety. ${ }^{15}$ The absolute configuration at C-3 was determined based on the trans relative configurations of the neighboring $\mathrm{H}-2$ and $\mathrm{H}-3$ protons. Based on the above spectroscopic data, the new compound, holstiinone A (1), was characterized as $\left(2 R, 2^{\prime \prime} R, 3 S, 3^{\prime \prime} S\right)-5^{\prime \prime}, 7$-dihydroxy-2,2" -bis( $4^{\prime}, 4^{\prime \prime \prime}$-hydroxyphenyl)-7" -methoxy-[3,3"-bichromane $]-4,4^{\prime \prime}$ dione.

Compound 2 was isolated as a white solid. The molecular formula $\mathrm{C}_{33} \mathrm{H}_{28} \mathrm{O}_{9}$ was assigned based on HRESIMS ([M + $\mathrm{H}]^{+} \mathrm{m} / z$ 569.1741, calcd 569.1812, Figure S16, Supporting Information) and NMR data (Table 1, Figures S9-S15, Supporting Information). It showed a specific rotation $[\alpha]^{24}$ of -92 (c $\left.0.013, \mathrm{CH}_{3} \mathrm{OH}\right)$. IR spectroscopy indicated the presence of hydroxy $\left(3320 \mathrm{~cm}^{-1}\right)$, conjugated carbonyl $(1698$ $\mathrm{cm}^{-1}$ ), and aromatic $\mathrm{C}=\mathrm{C}\left(1598\right.$ and $1515 \mathrm{~cm}^{-1}$ ) groups. The UV spectrum showed absorption maxima at 258 and 340 $\mathrm{nm}$, conforming the presence of a flavanone-type structure. ${ }^{26,29}$ The NMR spectroscopic data of 2 (Table 1, Figures S9-S15, Supporting Information) are closely related to those of compound 1. Compound 2 contained two additional methoxy groups with protons resonating at $\delta_{\mathrm{H}} 3.76\left(\delta_{\mathrm{C}} 55.8\right)$ and 3.78 $\left(\delta_{\mathrm{C}} 55.8\right)$ and attached to C-7 $\left(\delta_{\mathrm{C}} 165.1\right)$ and C- $4^{\prime \prime \prime}\left(\delta_{\mathrm{C}}\right.$ 155.7), respectively. For compound 2 , the attachments of the three methoxy groups at rings $A_{1}, A_{2}$, and $B_{2}$ were established based on the HMBC cross-peaks (Figure S13, Supporting Information $)$ of $\mathrm{H}_{3}-11\left(\delta_{\mathrm{H}} 3.76\right)$ and $\mathrm{H}-5\left(\delta_{\mathrm{H}} 7.66\right)$ to $\mathrm{C}-7\left(\delta_{\mathrm{C}}\right.$ $164.9)$; of $\mathrm{H}-11^{\prime}\left(\delta_{\mathrm{H}} 3.79\right)$, H-6" $\left(\delta_{\mathrm{H}} 6.14\right)$, and $\mathrm{H}-8^{\prime \prime}\left(\delta_{\mathrm{H}}\right.$ $6.11)$ to C-7" $\left(\delta_{\mathrm{C}} 166.4\right)$; and of H-11" $\left(\delta_{\mathrm{H}} 3.78\right)$ and $\mathrm{H}-2^{\prime \prime \prime} /$ $6^{\prime \prime \prime}\left(\delta_{\mathrm{H}} 7.36\right)$ to C-4"' $\left(\delta_{\mathrm{C}} 155.7\right)$. The 2,3-relative configuration of structure 2 was determined based on the ${ }^{1} \mathrm{H}$ NMR coupling constants and splitting patterns. The resonances assigned to $\mathrm{H}$ 2 and $\mathrm{H}-2^{\prime \prime}$ were doublets with a coupling constant of $10.2 \mathrm{~Hz}$ each, indicating 2,3- and 2",3"-trans relative configurations. The ECD spectrum of $\mathbf{2}$ (Figure 1) closely resembled that of $\mathbf{1}$ and was thus compatible with a $\left(2 R, 2^{\prime \prime} R, 3 S, 3^{\prime \prime} S\right)$ absolute configuration. Based on the above spectroscopic evidence, the new compound holstiinone B (2) was characterized as $\left(2 R, 2^{\prime \prime} R, 3 S, 3^{\prime \prime} S\right)-5^{\prime \prime}$-hydroxy-2-(4'-hydroxyphenyl)-7,7"'-dimethoxy-2" -(4"'-methoxyphenyl)-[3,3"-bichromane]-4,4"-dione. It is a methoxy derivative of $\mathbf{1}$, which is in agreement with the enzymatic $\mathrm{O}$-methylation, typical of flavonoids, by $\mathrm{O}$-methyltransferases. $^{30}$

Compound 3 was obtained as a pale yellow amorphous solid and was assigned the molecular formula $\mathrm{C}_{31} \mathrm{H}_{28} \mathrm{O}_{9}$ based on HRESIMS $\left([\mathrm{M}+\mathrm{Na}]^{+} \mathrm{m} / z\right.$ 567.1634, calcd 567.1631 (Figure S24, Supporting Information) and NMR data (Table 2, Figures S17-S23, Supporting Information). It showed a specific rotation $[\alpha]_{\mathrm{D}}^{24}$ of $-176.9\left(c 0.23, \mathrm{CH}_{3} \mathrm{OH}\right)$. IR absorptions at 3305,1700 , and $1598 \mathrm{~cm}^{-1}$ indicated the presence of hydroxy, conjugated carbonyl, and aromatic moieties, respectively. The UV spectrum showed absorption maxima at 340 and $264 \mathrm{~nm}$, corresponding to aromatic moieties of a flavanone. The ${ }^{1} \mathrm{H}$ NMR spectrum (Table 2; Figure S17, Supporting Information) showed 10 distinct aromatic proton signals. COSY and TOCSY correlations (Figures S19 and S23, Supporting Information) corroborated the presence of two $p$ disubstituted aromatic rings $\left(A_{1}\right.$ and $\left.B_{2}\right)$, two $A B X$ aromatic ring systems $\left(A_{2}\right.$ and $\left.B_{1}\right)$, and one tetrahydrofuran ring system, reminiscent of flavumchalcone, ${ }^{22}$ the only difference being the presence of a signal corresponding to a methoxy group, the protons of which resonate at $\delta_{\mathrm{H}} 3.28$. The attachment of the methoxy group to C-6" $6^{\prime \prime}\left(\delta_{\mathrm{C}} 83.6\right)$ was established based on the HMBC (Table 2, Figure S21, Supporting Information) cross-peaks of $\mathrm{H}_{3}-8\left(\delta_{\mathrm{H}} 3.28\right)$ to C-6" ${ }^{\prime \prime \prime}\left(\delta_{\mathrm{C}} 83.6\right)$. Further important $\mathrm{HMBC}$ cross-peaks that confirmed the molecular framework included those supporting the assignment of the tetrahydrofuran ring, namely, $\mathrm{H}-3^{\prime \prime \prime}\left(\delta_{\mathrm{H}} 4.69\right)$ to C-6" ${ }^{\prime \prime \prime}\left(\delta_{\mathrm{C}}\right.$ 83.6), C-1' $\left(\delta_{\mathrm{C}} 113.6\right)$, and C-5" ${ }^{\prime \prime \prime}\left(\delta_{\mathrm{C}} 81.3\right), \mathrm{H}-2^{\prime \prime} / 6^{\prime \prime}\left(\delta_{\mathrm{H}}\right.$ $6.94)$ to $\mathrm{C}-6^{\prime \prime \prime}\left(\delta_{\mathrm{C}} 57.0\right), \mathrm{H}-2^{\prime \prime \prime}\left(\delta_{\mathrm{H}} 3.22\right)$ to C-4" $4^{\prime \prime \prime}\left(\delta_{\mathrm{C}} 58.5\right)$ and $\mathrm{C}-7\left(\delta_{\mathrm{C}} 204.5\right)$, and $\mathrm{H}-4^{\prime \prime \prime}\left(\delta_{\mathrm{H}} 3.22\right)$ to C-1" $\left(\delta_{\mathrm{C}} 131.9\right)$, C-1"' $\left(\delta_{\mathrm{C}} 117.5\right)$, and C-7 $\left(\delta_{\mathrm{C}} 204.5\right)$. The placement of protons in ring $\mathrm{B}_{1}$ and $\mathrm{A}_{1}$ was supported by the $\mathrm{HMBC}$ crosspeak of H-6' $\left(\delta_{\mathrm{H}} 7.54\right)$ to C-7 $\left(\delta_{\mathrm{C}} 204.5\right)$ and H-2/6 $\left(\delta_{\mathrm{H}} 7.07\right)$ to $\mathrm{C}-2^{\prime \prime \prime}\left(\delta_{\mathrm{C}} 84.4\right)$, respectively. In addition, the protons of rings $\mathrm{A}_{2}$ and $\mathrm{B}_{2}$ were assigned based on the HMBC cross-peak of $\mathrm{H}-6^{\prime \prime \prime}\left(\delta_{\mathrm{H}} 7.02\right)$ to C-5" $5^{\prime \prime \prime}\left(\delta_{\mathrm{C}} 81.3\right)$ and that of $\mathrm{H}-2^{\prime \prime} / 6^{\prime \prime}\left(\delta_{\mathrm{H}}\right.$ 
Table 2. NMR Spectroscopic Data (500 MHz, Methanol- $d_{4}$ ) for Holstiichalcone I (3)

\begin{tabular}{|c|c|c|c|}
\hline position & $\delta_{\mathrm{C}}$, type & $\delta_{\mathrm{H}}(J$ in $\mathrm{Hz})$ & $\mathrm{HMBC}, \mathrm{H} \rightarrow \mathrm{C}$ \\
\hline 1 & $129.8, \mathrm{C}$ & & \\
\hline $2 / 6$ & 129.2, CH & 7.07, $\mathrm{AA}^{\prime}$ & C- $2^{\prime \prime \prime}, C-4, C-1$ \\
\hline $3 / 5$ & $115.4, \mathrm{CH}$ & 6.54, $\mathrm{XX}^{\prime}$ & C-1, C-4, C-2" \\
\hline 4 & 157.7, C & & \\
\hline $1^{\prime}$ & 113.6, C & & \\
\hline $2^{\prime}$ & $170.1, \mathrm{C}$ & & \\
\hline $3^{\prime}$ & 103.7, $\mathrm{CH}$ & $5.90, \mathrm{~d}(2.1)$ & $\mathrm{C}-5^{\prime}, \mathrm{C}-1^{\prime}, \mathrm{C}-4^{\prime}$ \\
\hline $4^{\prime}$ & 166.6, C & & \\
\hline $5^{\prime}$ & $110.4, \mathrm{CH}$ & 6.19, dd $(8.8,2.1)$ & $\mathrm{C}-1^{\prime}, \mathrm{C}-3^{\prime}, \mathrm{C}-4^{\prime}$ \\
\hline $6^{\prime}$ & $134.3, \mathrm{CH}$ & $7.54, \mathrm{~d}(9.0)$ & $\mathrm{C}-2^{\prime}, \mathrm{C}-4^{\prime}, \mathrm{C}-7$ \\
\hline $1^{\prime \prime}$ & $131.9, \mathrm{C}$ & & \\
\hline $2^{\prime \prime} / 6^{\prime \prime}$ & $128.9, \mathrm{CH}$ & 6.94, $\mathrm{AA}^{\prime}$ & $\mathrm{C}-4^{\prime \prime}, \mathrm{C}-6^{\prime \prime \prime}, \mathrm{C}-1^{\prime \prime}$ \\
\hline $3^{\prime \prime} / 5^{\prime \prime}$ & $116.0, \mathrm{CH}$ & $6.51, \mathrm{XX}^{\prime}$ & $C-1^{\prime \prime}, C-4^{\prime \prime}, C-5^{\prime \prime}$ \\
\hline $4^{\prime \prime}$ & $157.9, \mathrm{C}$ & & \\
\hline $1^{\prime \prime \prime}$ & $117.5, \mathrm{C}$ & & \\
\hline $2^{\prime \prime \prime}$ & 157.6, C & & \\
\hline $3^{\prime \prime \prime}$ & 103.9, $\mathrm{CH}$ & $6.31, \mathrm{~d}(2.1)$ & $C-5^{\prime \prime \prime}, C-4^{\prime \prime \prime}, C-2^{\prime \prime \prime}$ \\
\hline $4^{\prime \prime \prime}$ & $159.4, \mathrm{C}$ & & \\
\hline $5^{\prime \prime \prime}$ & $107.8, \mathrm{CH}$ & 6.26 , dd $(8.2,2.1)$ & C- $1^{\prime \prime \prime}, C-3^{\prime \prime \prime}, C-4^{\prime \prime \prime}$ \\
\hline $6^{\prime \prime \prime}$ & 131.4, $\mathrm{CH}$ & $7.02, \mathrm{~d}(8.2)$ & $\mathrm{C}-2^{\prime \prime \prime}, \mathrm{C}-4^{\prime \prime \prime}, \mathrm{C}-5^{\prime \prime \prime \prime}$ \\
\hline $2^{\prime \prime \prime}$ & $84.4, \mathrm{CH}$ & $5.27, \mathrm{~d}(8.2)$ & $\mathrm{C}-2, \mathrm{C}-3^{\prime \prime \prime}, \mathrm{C}-6, \mathrm{C}-7$ \\
\hline $3^{\prime \prime \prime}$ & $52.9, \mathrm{CH}$ & 4.69, dd $(8.2,5.5)$ & $\mathrm{C}-2^{\prime \prime \prime}, \mathrm{C}-4^{\prime \prime \prime}, \mathrm{C}-1^{\prime}, \mathrm{C}-7$ \\
\hline $4^{\prime \prime \prime}$ & $58.5, \mathrm{CH}$ & $\begin{array}{l}3.22, \text { ddd }(9.2,5.5 \text {, } \\
5.4)\end{array}$ & $\begin{array}{l}\mathrm{C}-3^{\prime \prime \prime}, \mathrm{C}-1^{\prime \prime}, \mathrm{C}-5^{\prime \prime \prime}, \mathrm{C}-6^{\prime \prime \prime}, \\
\text {, }\end{array}$ \\
\hline $5^{\prime \prime \prime}$ & 81.3, $\mathrm{CH}$ & $5.12, \mathrm{~d}(9.2)$ & $\mathrm{C}-4^{\prime \prime \prime}, \mathrm{C}-6^{\prime \prime \prime}, \mathrm{C}-2^{\prime \prime \prime}$ \\
\hline $6^{\prime \prime \prime}$ & 83.6, $\mathrm{CH}$ & $4.27, \mathrm{~d}(5.4)$ & $\begin{array}{l}\mathrm{C}-2^{\prime \prime}, \mathrm{C}-3^{\prime \prime \prime}, \mathrm{C}-4^{\prime \prime \prime}, \mathrm{C}-5^{\prime \prime \prime}, \\
\mathrm{C}-6^{\prime \prime}, \mathrm{C}-8\end{array}$ \\
\hline 7 & 204.5, C & & \\
\hline 8 & $57.0, \mathrm{CH}$ & $3.28, \mathrm{~s}$ & $\mathrm{C}-6^{\prime \prime \prime}$ \\
\hline
\end{tabular}

6.94) to C-6" ${ }^{\prime \prime \prime}\left(\delta_{\mathrm{C}} 57.0\right)$, respectively (Table 2, Figure S21, Supporting Information).

The protons belonging to the spin system of the tetrahydrofuran ring were assigned based on the COSY and TOCSY correlations (Figure S19 and S22, Supporting Information) of $\mathrm{H}-2^{\prime \prime \prime}\left(\delta_{\mathrm{H}} 5.27\right)$ to $\mathrm{H}-3^{\prime \prime \prime}\left(\delta_{\mathrm{H}} 4.69\right), \mathrm{H}-3^{\prime \prime \prime \prime}$ $\left(\delta_{\mathrm{H}} 4.69\right)$ to $\mathrm{H}-4^{\prime \prime \prime}\left(\delta_{\mathrm{H}} 3.22\right)$, and H-4" ${ }^{\prime \prime}\left(\delta_{\mathrm{H}} 3.22\right)$ to H-5" $\left(\delta_{\mathrm{H}} 5.12\right)$ and $\mathrm{H}-6^{\prime \prime \prime}\left(\delta_{\mathrm{H}} 4.27\right)$. The relative configurations at the carbon atoms of the tetrahydrofuran ring were assigned based on the NOESY correlations (Figure 2) of $\mathrm{H}-2^{\prime \prime \prime \prime}\left(\delta_{\mathrm{H}}\right.$
5.27) and H-5" $5^{\prime \prime \prime}\left(\delta_{\mathrm{H}} 5.12\right), \mathrm{H}-2^{\prime \prime \prime}\left(\delta_{\mathrm{H}} 5.27\right)$ and H-3"' $\left(\delta_{\mathrm{H}}\right.$ 4.69), H-5" $5^{\prime \prime \prime}\left(\delta_{\mathrm{H}} 5.12\right)$ and H-6"' $\left(\delta_{\mathrm{H}} 4.27\right)$, and H-4" ${ }^{\prime \prime \prime}\left(\delta_{\mathrm{H}}\right.$ $3.22)$ with $\mathrm{H}-6^{\prime \prime \prime}\left(\delta_{\mathrm{H}} 4.27\right)$. Based on the above spectroscopic data, the new compound, holstiichalcone I (3), was characterized as (2'"',4"'-dihydroxyphenyl)$\left[\left(2^{\prime \prime \prime} S^{*}, 3^{\prime \prime \prime} R^{*}, 4^{\prime \prime \prime} S^{*}, 5^{\prime \prime \prime} R^{*}\right)-5^{\prime \prime \prime}-\left(2^{\prime}, 4^{\prime}\right.\right.$-dihydroxyphenyl)$2^{\prime \prime \prime}$-(4-hydroxyphenyl)-4" $4^{\prime \prime}-\left\{\left(4^{\prime \prime}\right.\right.$-hydroxyphenyl)(methoxy)methyl $\}$ tetrahydrofuran-3" $3^{\prime \prime}$-yl]methanone. Despite repeated attempts, no crystals suitable for X-ray crystallography to determine the absolute configuration of 3 were obtained. The ECD spectrum is shown in Figure 1. Similar structures have previously been reported from various Ochna, ${ }^{27,31,32}$ Campylospermum, ${ }^{22,33}$ and Lophira ${ }^{34,35}$ species, however without their absolute configuration having been determined.

Compound $\mathbf{4}$ was isolated as a yellow solid and was assigned the molecular formula $\mathrm{C}_{30} \mathrm{H}_{22} \mathrm{O}_{8}$ based on HRESIMS ([ $\mathrm{M}+$ $\mathrm{H}]^{+} m / z$ 511.1478, calcd 511.1393, Figure S32, Supporting Information) and NMR data (Table 3, Figures S25-S31, Supporting Information). It showed a specific rotation $[\alpha]_{D}^{24}$ of -21 (c $\left.0.013, \mathrm{CH}_{3} \mathrm{OH}\right)$. IR vibrations at 3382 and 1604 $\mathrm{cm}^{-1}$ suggested the presence of hydroxy and $\mathrm{C}=\mathrm{C}$ groups, respectively. The UV spectrum displayed absorption bands centered at 368,340 , and $284 \mathrm{~nm}$ that were in agreement with a chalcone skeleton. ${ }^{7}$ The ${ }^{13} \mathrm{C}$ NMR spectrum (Table 3, Figure S26, Supporting Information) showed only 15 instead of 30 resonances, revealing compound 4 to be a symmetric dimeric molecule. The ${ }^{1} \mathrm{H}$ NMR data (Table 3, Figures S25 and S27, Supporting Information) indicated the presence of two distinct $\mathrm{ABX}$ spin systems at $\delta_{\mathrm{H}} 7.99\left(\mathrm{H}-6^{\prime} / 6^{\prime \prime \prime}\right), 6.42\left(\mathrm{H}-5^{\prime} / 5^{\prime \prime \prime}\right)$, and $6.30\left(\mathrm{H}-3^{\prime} / 3^{\prime \prime \prime}\right)$ and at $7.70\left(\mathrm{H}-4^{\prime \prime} / 4^{\prime \prime \prime \prime}\right), 7.68\left(\mathrm{H}-6^{\prime \prime} / 6^{\prime \prime \prime \prime}\right)$, and $7.01\left(\mathrm{H}-3^{\prime \prime} / 3^{\prime \prime \prime \prime}\right)$. In addition, trans olefinic protons resonating at $\delta_{\mathrm{H}} 7.88\left(\mathrm{H}-3 / 3^{\prime \prime \prime}\right)$ and $7.67\left(\mathrm{H}-2 / 2^{\prime \prime \prime}\right)$ suggested the presence of a chalcone moiety. Furthermore, the ${ }^{13} \mathrm{C}$ NMR spectrum (Table 3, Figure S26, Supporting Information) consisted of signals due to a carbonyl function at $\delta_{\mathrm{C}} 193.6$ (C$\left.1 / 1^{\prime \prime \prime}\right)$, three oxygenated aromatic carbons at $\delta_{\mathrm{C}} 167.5$ (C-2'/ $\left.2^{\prime \prime \prime \prime}\right), 166.4\left(\mathrm{C}-4^{\prime} / 4^{\prime \prime \prime \prime}\right)$, and $158.9\left(\mathrm{C}-2^{\prime \prime} / 2^{\prime \prime \prime \prime}\right)$, and 11 other $\mathrm{sp}^{2}$-hybridized carbons between 145.6 and $103.8 \mathrm{ppm}$. The locations of the trans olefinic systems and the ABX spin systems were corroborated by HMBC cross-peaks (Table 3, Figure S29, Supporting Information) of H-6'/6"'"' $\left(\delta_{\mathrm{H}} 7.99\right)$ and $\mathrm{H}-3 / 3^{\prime \prime \prime \prime}\left(\delta_{\mathrm{H}} 7.88\right)$ to $\mathrm{C}-1 / 1^{\prime \prime \prime}\left(\delta_{\mathrm{C}} 193.6\right)$ and of $\mathrm{H}-2 / 2^{\prime \prime \prime}$ $\left(\delta_{\mathrm{H}} 7.67\right)$ and $\mathrm{H}-3^{\prime \prime} / 3^{\prime \prime \prime \prime}(7.01)$ to $\mathrm{C}-1^{\prime \prime} / 1^{\prime \prime \prime \prime}\left(\delta_{\mathrm{C}} 128.2\right)$. In a

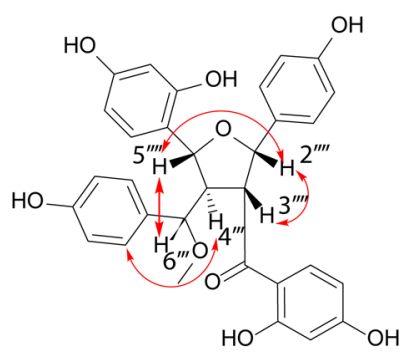

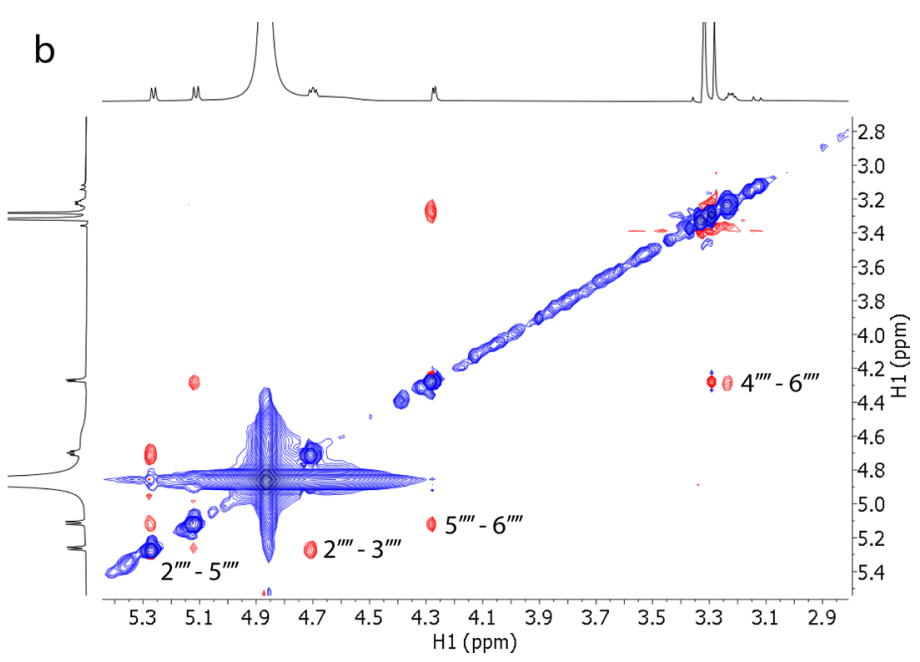

Figure 2. Key NOE correlations allowing the determination of the relative configuration of the tetrahydrofuran ring of holstiichalcone I (3). 
Table 3. NMR Spectroscopic Data (500 MHz, Methanol- $d_{4}$ ) for Holstiichalcone II (4)

\begin{tabular}{|c|c|c|c|}
\hline position & $\delta_{\mathrm{C}}$, type & $\delta_{\mathrm{H}}(J$ in $\mathrm{Hz})$ & $\mathrm{HMBC}, \mathrm{H} \rightarrow \mathrm{C}$ \\
\hline 1 & 193.6, C & & \\
\hline 2 & $118.7, \mathrm{CH}$ & 7.67, m & $\mathrm{C}-1, \mathrm{C}-5^{\prime \prime}, \mathrm{C}-1^{\prime}$ \\
\hline 3 & 145.6, $\mathrm{CH}$ & $7.88, \mathrm{~d}(15.3)$ & C-1, C- $4^{\prime \prime}$, C-6" \\
\hline $1^{\prime}$ & 114.7, C & & \\
\hline $2^{\prime}$ & 167.5, C & & \\
\hline $3^{\prime}$ & $103.8, \mathrm{CH}$ & $6.30, \mathrm{~d}(2.3)$ & $\mathrm{C}-1^{\prime}, \mathrm{C}-5^{\prime}$ \\
\hline $4^{\prime}$ & $166.4, \mathrm{C}$ & & \\
\hline $5^{\prime}$ & $109.2, \mathrm{CH}$ & $6.42, \mathrm{~d}(2.4)$ & $\mathrm{C}-3^{\prime}, \mathrm{C}-1^{\prime}$ \\
\hline $6^{\prime}$ & 133.5, $\mathrm{CH}$ & $7.99, \mathrm{~d}(8.8)$ & $\mathrm{C}-1, \mathrm{C}-2^{\prime}, \mathrm{C}-4^{\prime}$ \\
\hline $1^{\prime \prime}$ & 128.2, C & & \\
\hline $2^{\prime \prime}$ & $158.9, \mathrm{C}$ & & \\
\hline $3^{\prime \prime}$ & $117.6, \mathrm{C}$ & $7.01, \mathrm{~d}(8.8)$ & $\mathrm{C}-1^{\prime \prime}, \mathrm{C} 5^{\prime \prime}$ \\
\hline $4^{\prime \prime}$ & $130.8, \mathrm{CH}$ & $7.70, \mathrm{~m}$ & C-3, C-2", C-6" \\
\hline $5^{\prime \prime}$ & 127.5, C & & \\
\hline $6^{\prime \prime}$ & $133.8, \mathrm{CH}$ & $7.68, \mathrm{~m}$ & $\mathrm{C}-2^{\prime \prime}, \mathrm{C}-4^{\prime \prime}$ \\
\hline $1^{\prime \prime \prime}$ & 193.6, C & & \\
\hline $2^{\prime \prime \prime}$ & 118.7, CH & $7.67, \mathrm{~m}$ & $\mathrm{C}-1^{\prime \prime \prime}, \mathrm{C}-4^{\prime \prime \prime \prime \prime}, \mathrm{C}-5^{\prime \prime \prime \prime \prime}$ \\
\hline $3^{\prime \prime \prime}$ & 145.6, $\mathrm{CH}$ & $6.42, \mathrm{~d}(15.3)$ & $\mathrm{C}-1^{\prime \prime \prime}, \mathrm{C}-4^{\prime \prime \prime \prime \prime}, \mathrm{C}-6^{\prime \prime \prime \prime \prime}$ \\
\hline $1^{\prime \prime \prime \prime}$ & 114.7, C & & \\
\hline $2^{\prime \prime \prime}$ & 167.5, C & & \\
\hline $3^{\prime \prime \prime \prime}$ & $103.8, \mathrm{CH}$ & $6.30, \mathrm{~d}(2.3)$ & $C-1^{\prime \prime \prime}, \mathrm{C}-5^{\prime \prime \prime \prime}$ \\
\hline $4^{\prime \prime \prime \prime}$ & 166.4, C & & \\
\hline $5^{\prime \prime \prime}$ & 109.2, $\mathrm{CH}$ & $6.42, \mathrm{~d}(2.4)$ & $\mathrm{C}-3^{\prime \prime \prime \prime}, \mathrm{C}-1^{\prime \prime \prime \prime}$ \\
\hline $6^{\prime \prime \prime}$ & 133.5, $\mathrm{CH}$ & & $\mathrm{C}-1^{\prime \prime \prime}, \mathrm{C}-2^{\prime \prime \prime \prime}, \mathrm{C}-4^{\prime \prime \prime}$ \\
\hline $1^{\prime \prime \prime \prime \prime}$ & 128.2, C & & \\
\hline $2^{\prime \prime \prime \prime \prime}$ & $158.9, \mathrm{C}$ & & \\
\hline $3^{\prime \prime \prime \prime \prime}$ & 117.6, C & $7.01, \mathrm{~d}(8.8)$ & \\
\hline $4^{\prime \prime \prime \prime \prime}$ & $130.8, \mathrm{CH}$ & $7.70, \mathrm{~m}$ & C- $3^{\prime \prime \prime}, C-2^{\prime \prime \prime \prime \prime}, C-6^{\prime \prime \prime \prime}$ \\
\hline $5^{\prime \prime \prime \prime \prime}$ & 127.5, C & & \\
\hline $6^{\prime \prime \prime \prime \prime}$ & $133.8, \mathrm{CH}$ & $7.68, \mathrm{~m}$ & $\mathrm{C}-2^{\prime \prime \prime \prime \prime}, \mathrm{C}-4^{\prime \prime \prime \prime \prime}$ \\
\hline
\end{tabular}

addition, the HMBC cross-peaks of $\mathrm{H}-6^{\prime} / 6^{\prime \prime \prime}\left(\delta_{\mathrm{H}} 7.99\right)$ to C$1 / 1^{\prime \prime \prime}\left(\delta_{\mathrm{C}} 193.6\right), \mathrm{C}-4^{\prime} / 4^{\prime \prime \prime}\left(\delta_{\mathrm{C}} 166.4\right)$, and C-2 $2^{\prime} / 2^{\prime \prime \prime}\left(\delta_{\mathrm{C}}\right.$ $167.5)$ and of $\mathrm{H}-3 / 3^{\prime \prime \prime}\left(\delta_{\mathrm{H}} 7.88\right)$ to $\mathrm{C}-1 / 1^{\prime \prime \prime}\left(\delta_{\mathrm{C}} 193.6\right)$ and C$4^{\prime \prime} / 4^{\prime \prime \prime}\left(\delta_{\mathrm{C}} 130.8\right)$ (Table 3, Figure S29, Supporting Information) confirmed the connection of the ABX rings $A_{1} /$ $\mathrm{A}_{2}$ and trans olefinic units to carbonyl groups. Furthermore, the common HMBC cross-peak of $\mathrm{H}-3^{\prime \prime} / 3^{\prime \prime \prime \prime}\left(\delta_{\mathrm{H}} 7.01\right)$ and $\mathrm{H}-2 / 2^{\prime \prime \prime}\left(\delta_{\mathrm{H}} 7.67\right)$ to C-5" $/ 5^{\prime \prime \prime \prime \prime}\left(\delta_{\mathrm{C}} 127.5\right)$ (Table 3, Figure S29, Supporting Information $)$ and $\mathrm{H}-3 / 3^{\prime \prime \prime \prime}\left(\delta_{\mathrm{H}} 7.01\right)$ to C$1^{\prime \prime} / 1^{\prime \prime \prime \prime \prime}\left(\delta_{\mathrm{C}} 128.2\right)$ supported the assignment of rings $\mathrm{B}_{1} / \mathrm{B}_{2}$. Compound 4 exhibited optical activity because of its axis of chirality (atropisomerism), supporting its dimeric nature, which resembles previously reported dimeric chalconoids. ${ }^{7,36-39}$ The ECD spectrum (Figure 1) shows positive Cotton effects at 204, 253,293, and $436 \mathrm{~nm}$ and negative Cotton effects at 230, 274, 320, and $373 \mathrm{~nm}$. As there are no published ECD spectra available for bichalcones with known absolute configuration, the ECD of the related biflavones may provide a handle on the structure determination of 4 . Hence the spectrum of 4 shows resemblance to the ECD spectrum of $(-)-\left(S_{\mathrm{a}}\right)$-agatisflavone, ${ }^{36}$ whereas little to none to that of $(-)-\left(R_{\mathrm{a}}\right)-4^{\prime}, 4^{\prime \prime}, 7,7^{\prime \prime}$-tetra-O-methylcupressuflavone. ${ }^{40}$ Its negative specific rotation $\left([\alpha]^{24}\right.$ of -21$)$ and negative Cotton effects at 373 and $320 \mathrm{~nm}$ therefore suggest that 4 most likely has an $\left(S_{\mathrm{a}}\right)$-bichalcone unit and thus a $P$-configuration. ${ }^{41,42}$ Based on the above spectroscopic features, the new compound holstiichalcone II (4) was characterized as $(-)-\left(S_{\mathrm{a}}\right)-\left(2 E, 2^{\prime \prime \prime} E\right)$ $3,3^{\prime \prime \prime}-\left(2^{\prime \prime}, 2^{\prime \prime \prime \prime}\right.$-dihydroxy-[1,1'"'-biphenyl $]-3,3^{\prime \prime \prime}$-diyl $)$ bis $[1$ - (2',4'-dihydroxyphenyl)prop-2-en-1-one]. Similar compounds have been previously reported from Rhus pyroides. ${ }^{7}$

Compound 5 was isolated as a yellow solid and was assigned the molecular formula $\mathrm{C}_{30} \mathrm{H}_{24} \mathrm{O}_{8}$ based on HRESIMS ([M + $\mathrm{H}]^{+} m / z$ 513.1575, calcd 513.1549, Figure S40, Supporting Information) and NMR data (Table 4, Figures S33-S39,

Table 4. NMR Spectroscopic Data (500 MHz, Methanol- $d_{4}$ ) for Holstiichalcone III (5)

\begin{tabular}{|c|c|c|c|}
\hline position & $\delta_{\mathrm{C}}$, type & $\delta_{\mathrm{H}}(J$ in $\mathrm{Hz})$ & $\mathrm{HMBC}, \mathrm{H} \rightarrow \mathrm{C}$ \\
\hline 1 & 193.3, C & & \\
\hline 2 & 119.4, $\mathrm{CH}$ & 7.57, d (15.4) & $\mathrm{C}-2^{\prime}, \mathrm{C}-2 \mathrm{C}-6^{\prime}, \mathrm{C}-1$ \\
\hline 3 & $144.9, \mathrm{CH}$ & $7.72, \mathrm{~d}(15.4)$ & $\mathrm{C}-3, \mathrm{C}-1^{\prime}, \mathrm{C}-1, \mathrm{C}-4^{\prime \prime}$ \\
\hline $1^{\prime}$ & 114.6, C & & \\
\hline $2^{\prime}$ & 166.6, C, & & \\
\hline $3^{\prime}$ & $103.8, \mathrm{CH}$ & 6.27, $\mathrm{AA}^{\prime}$ & $\mathrm{C}-1^{\prime}, \mathrm{C}-5^{\prime}$ \\
\hline $4^{\prime}$ & 167.5, C & & \\
\hline $5^{\prime}$ & 109.4, C & $6.38, \mathrm{dd}(8.8,2.4)$ & \\
\hline $6^{\prime}$ & 133.4, $\mathrm{CH}$ & 7.91, $\mathrm{XX}^{\prime}$ & $\mathrm{C}-1, \mathrm{C}-2^{\prime}, \mathrm{C}-4^{\prime}$ \\
\hline $1^{\prime \prime}$ & 128.7, C & & \\
\hline $2^{\prime \prime}$ & 122.1, $\mathrm{CH}$ & $7.31, \mathrm{~d}(2.4)$ & $\mathrm{C}-6^{\prime \prime}, \mathrm{C}-4^{\prime \prime}$ \\
\hline $3^{\prime \prime}$ & 145.7, C & & \\
\hline $4^{\prime \prime}$ & $153.3, \mathrm{C}$ & & \\
\hline $5^{\prime \prime}$ & $118.5, \mathrm{CH}$ & $7.00 \mathrm{~d}(8.8)$ & $\mathrm{C}-1^{\prime \prime}, \mathrm{C}-3^{\prime \prime}$ \\
\hline $6^{\prime \prime}$ & $127.4, \mathrm{CH}$ & $7.46, \mathrm{dd}(8.8,2.4)$ & C-2", C-4" \\
\hline $1^{\prime \prime \prime}$ & 205.2, C & & \\
\hline $2^{\prime \prime \prime}$ & $40.6, \mathrm{CH}_{2}$ & $3.24, \mathrm{t}(7.6)$ & $\mathrm{C}-1^{\prime \prime \prime}, \mathrm{C}-4^{\prime \prime \prime \prime \prime}, \mathrm{C}-3^{\prime \prime \prime}, \mathrm{C}-1^{\prime \prime \prime}$ \\
\hline $3^{\prime \prime \prime}$ & $31.0, \mathrm{CH}_{2}$ & $3.00, \mathrm{t}(7.6)$ & $\mathrm{C}-3^{\prime \prime \prime \prime} / 5^{\prime \prime \prime \prime}, \mathrm{C}-2^{\prime \prime \prime}, \mathrm{C}-1^{\prime \prime \prime}$ \\
\hline $1^{\prime \prime \prime}$ & $114.0, \mathrm{C}$ & & \\
\hline $2^{\prime \prime \prime \prime}$ & 166.4 & & \\
\hline $3^{\prime \prime \prime \prime}$ & 103.7, $\mathrm{CH}$ & $6.24, \mathrm{~d}(2.4)$ & $\mathrm{C}-1^{\prime \prime \prime}, \mathrm{C}-5^{\prime \prime \prime \prime}$ \\
\hline $4^{\prime \prime \prime}$ & $166.8, \mathrm{C}$ & & \\
\hline $5^{\prime \prime \prime \prime}$ & 109.3, CH & 6.26 , dd $(8.8,2.4)$ & $\mathrm{C}-3^{\prime \prime \prime}, \mathrm{C}-1^{\prime \prime \prime}$ \\
\hline $6^{\prime \prime \prime \prime}$ & $133, \mathrm{CH}$ & 7.73, dd $(8.8)$ & $\mathrm{C}-2^{\prime \prime \prime}, \mathrm{C}-2^{\prime \prime \prime}, \mathrm{C}-1^{\prime \prime \prime}$ \\
\hline $1^{\prime \prime \prime \prime \prime}$ & $157.6, \mathrm{C}$ & & \\
\hline $2^{\prime \prime \prime \prime \prime} / 6^{\prime \prime \prime \prime \prime}$ & 118.3, $\mathrm{CH}$ & $6.89, \mathrm{~d}(8.6)$ & $\mathrm{C}-3^{\prime \prime \prime \prime \prime} / 5^{\prime \prime \prime \prime}, \mathrm{C}-1^{\prime \prime \prime \prime}, \mathrm{C}-4^{\prime \prime \prime \prime}$ \\
\hline $3^{\prime \prime \prime \prime} / 5^{\prime \prime \prime \prime}$ & $130.7, \mathrm{CH}$ & $7.23, \mathrm{~d}(8.6)$ & $\mathrm{C}-2^{\prime \prime \prime \prime \prime} / 6^{\prime \prime \prime \prime \prime}, \mathrm{C}-1^{\prime \prime \prime \prime \prime}, \mathrm{C}-3^{\prime \prime \prime}$ \\
\hline $4^{\prime \prime \prime \prime \prime}$ & 136.9, C & & \\
\hline
\end{tabular}

Supporting Information). IR absorptions at 3326 and 1709 $\mathrm{cm}^{-1}$ indicated the presence of hydroxy and conjugated carbonyl moieties, respectively. IR absorptions at 1631 and $1506 \mathrm{~cm}^{-1}$ are typical of $\mathrm{C}=\mathrm{C}$ stretches. The UV spectrum with absorption maxima centered at $\lambda_{\max } 264,320$, and $346 \mathrm{~nm}$ indicated the presence of a chalconoid skeleton. ${ }^{29,43}$ The ${ }^{1} \mathrm{H}$ NMR spectrum (Table 4, Figure S33, Supporting Information) displayed signals of a $p$-disubstituted aromatic ring resonating at $\delta_{\mathrm{H}} 7.23\left(\mathrm{H}-3^{\prime \prime \prime \prime} / 5^{\prime \prime \prime \prime}\right)$ and $6.89\left(\mathrm{H}-2^{\prime \prime \prime \prime \prime} / 6^{\prime \prime \prime \prime \prime}\right)$, three sets of $\mathrm{ABX}$ spin systems at $7.73\left(\mathrm{H}-6^{\prime \prime \prime}\right), 6.24\left(\mathrm{H}-3^{\prime \prime \prime}\right)$, and $6.26(\mathrm{H}-$ $\left.5^{\prime \prime \prime}\right)$ for ring A, at $\delta_{\mathrm{H}} 7.31\left(\mathrm{H}-2^{\prime \prime}\right), 7.00\left(\mathrm{H}-5^{\prime \prime}\right)$, and $7.46(\mathrm{H}-$ $\left.6^{\prime \prime}\right)$ for ring $\mathrm{B}_{2}$, and at $7.91\left(\mathrm{H}-6^{\prime}\right), 6.38\left(\mathrm{H}-5^{\prime}\right)$, and $6.27(\mathrm{H}-$ $\left.3^{\prime}\right)$ for ring $A_{2}$. The ${ }^{1} \mathrm{H}$ NMR spectrum consisted also of signals corresponding to trans-olefinic protons at $\delta_{\mathrm{H}} 7.72(\mathrm{H}$ $3)$ and $7.57(\mathrm{H}-2)$ and ethylene protons at $\delta_{\mathrm{H}} 3.24\left(\mathrm{H}-2^{\prime \prime \prime}\right)$ and $3.00\left(\mathrm{H}-3^{\prime \prime \prime}\right)$, which are common features of chalcone and dihydrochalcone moieties, respectively. The ${ }^{13} \mathrm{C}$ NMR spectrum (Table 4, Figure S34, Supporting Information) of 5 showed a total of 30 carbons comprising two carbonyl carbons at $\delta_{\mathrm{C}} 193.3(\mathrm{C}-1)$ and $205.3\left(\mathrm{C}-1^{\prime \prime \prime}\right)$, six oxygenated aromatic carbons (167.6-145.7 ppm), $20 \mathrm{sp}^{2}$-hybridized carbons (144.9-103.7 ppm), and two methylene carbons at $\delta_{\mathrm{C}} 40.6\left(\mathrm{C}-2^{\prime \prime \prime}\right)$ and $31.0\left(\mathrm{C}-3^{\prime \prime \prime}\right)$. Analysis of the HMBC 
(Figure S37, Supporting Information) cross-peaks of $\mathrm{H}-2^{\prime \prime \prime}\left(\delta_{\mathrm{H}}\right.$ $3.24), \mathrm{H}-3^{\prime \prime \prime}\left(\delta_{\mathrm{H}} 3.00\right)$, and H-6"' $\left(\delta_{\mathrm{H}} 7.73\right)$ to C-1"' $\left(\delta_{\mathrm{C}}\right.$, $205.2)$ and that of $\mathrm{H}-2^{\prime \prime \prime}\left(\delta_{\mathrm{H}} 3.24\right), \mathrm{H}-3^{\prime \prime \prime}\left(\delta_{\mathrm{H}} 3.00\right), \mathrm{H}-2^{\prime \prime \prime \prime \prime} /$ $6^{\prime \prime \prime \prime \prime}\left(\delta_{\mathrm{H}} 6.89\right)$, and $\mathrm{H}-3^{\prime \prime \prime \prime} / 5^{\prime \prime \prime \prime}\left(\delta_{\mathrm{H}} 7.23\right)$ to C-1"'"' $\left(\delta_{\mathrm{C}}\right.$ $157.6)$ allowed the assignment of the dihydrochalcone moiety possessing rings $\mathrm{A}$ and $\mathrm{B}$. In addition, the $\mathrm{HMBC}$ cross-peaks of H-3 $\left(\delta_{\mathrm{H}} 7.72\right), \mathrm{H}-2\left(\delta_{\mathrm{H}} 7.57\right)$, and $\mathrm{H}-6^{\prime}\left(\delta_{\mathrm{H}} 7.91\right)$ to C-1 $\left(\delta_{\mathrm{C}} 193.3\right)$ and that of H-5" $\left(\delta_{\mathrm{H}} 7.00\right)$ to C-1" ${ }^{\prime \prime}\left(\delta_{\mathrm{C}} 128.7\right)$ and C-3" $\left(\delta_{\mathrm{C}} 145.7\right)$ and H-6" $\left(\delta_{\mathrm{H}} 7.46\right)$ to C-3 $\left(\delta_{\mathrm{C}} 144.9\right)$ allowed the assignment of the chalcone moiety possessing ring $\mathrm{A}_{2}$ and ring $\mathrm{B}_{2}$. The NOESY correlation of $\mathrm{H}-2^{\prime \prime}\left(\delta_{\mathrm{H}} 7.31\right)$ to $\mathrm{H}-2^{\prime \prime \prime \prime \prime} /$ $6^{\prime \prime \prime \prime \prime}\left(\delta_{\mathrm{H}} 6.89\right)$ corroborated the connectivity of the chalcone and dihydrochalcone moieties. Based on the above spectroscopic analyses, the new compound holstiichalcone III (5) was characterized as (E)-1-(2',4'-dihydroxyphenyl)-3- $\left(3^{\prime \prime}-\left[4^{\prime \prime \prime}\right.\right.$ $\left\{1^{\prime \prime \prime}-\left(2^{\prime \prime \prime}, 4^{\prime \prime \prime}\right.\right.$-dihydroxyphenyl $)-5^{\prime \prime \prime \prime \prime}$-oxopropyl $\}$ phenoxy $]-4^{\prime \prime}$ hydroxyphenyl)prop-2'-en-1-one. Similar compounds constituting chalcone and dihydrochalcone moieties have been reported from Rhus pyroides, ${ }^{7}$ Luxemburgia octandra, ${ }^{44}$ and Schinopsis brasiliensis. ${ }^{45}$

In addition to the five new compounds, 11 known flavonoid analogues, 6-16 (Figures S41-S84, Supporting Information), were isolated from the stem bark of $O$. holstii. Previously related secondary metabolites have also been reported from the Thymelaeaceae and Anacardiceae families. ${ }^{16,17,28,46-48,7,36,44,45,49}$ Such interfamily phytochemical similarities are indicative of phylogenetic relationships and imply that the plant families possess similar enzymatic systems.

The crude extract of the stem bark of $O$. holstii and its constituents were evaluated for antibacterial activity against $B$. subtilis (Gram-positive) and E. coli (Gram-negative), as well as for cytotoxicity against the MCF-7 human breast cancer cell line (Figures S85-S88, Table 5, Supporting Information). The crude extract exhibited significant activity against $B$. subtilis with an MIC value of $9.1 \mu \mathrm{g} / \mathrm{mL}$. Holstiinone A (1) showed moderate antibacterial activity $(\mathrm{MIC}=14.0 \mu \mathrm{M})$, whereas the other tested compounds showed no significant activity (MIC > $250 \mu \mathrm{M})$ against $B$. subtilis. Furthermore, none of the investigated compounds showed substantial antibacterial activity against E. coli. Antibacterial activity dose-response curves for compounds 1, 5, 6, 8, 9, and 14 against Grampositive B. subtilis and that of compound 14 against Gramnegative E. coli are summarized in Figure S87 (Supporting Information). The compounds were also tested for cytotoxicity against the MCF-7 human breast cancer cell lines. Most of these did not show substantial cytotoxicity $\left(\mathrm{EC}_{50} \geq 350 \mu \mathrm{M}\right)$, while the crude extract and lophirone F (14) exhibited moderate cytotoxicity with $\mathrm{EC}_{50}$ values of $11 \mu \mathrm{g} / \mathrm{mL}$ and 24 $\mu \mathrm{M}$, respectively (Figure S88, Supporting Information). Previous pharmacological studies have indicated that biflavanones and chalconoids possess antibacterial, ${ }^{18}$ antimalarial, ${ }^{6,50}$ anti-inflammatory, ${ }^{15}$ cytotoxic, ${ }^{47}$ anti-HIV, ${ }^{51}$ and antifungal ${ }^{28}$ activities, indicating their broad spectrum of biomedical potential, warranting further exploration of the genus Ochna and the Ochnaceae family in general for drug discovery. Antibacterial activity of compounds 1, 5, 6, 8, 9, and 14 against Gram-positive B. subtilis and $\mathbf{1 4}$ against Gram-negative E. coli, showing the $\mathrm{EC}_{50}, \mathrm{EC}_{90}$, and $\mathrm{MIC}$ values (in $\mu \mathrm{M}$ ), is summarized in Table S5 (Supporting Information).

In conclusion, five new natural products $(\mathbf{1}-\mathbf{5})$ were isolated from the stem bark extract of $O$. holstii along with 11 known compounds. The biflavanones $(1,2,6-10)$ and bichalconoids $(3,4,5,15,16)$ isolated from this species are in line with the richness of the Ochnaceae family in this type of compounds. The compounds also highlight the chemotaxonomic relationship of the Ochnaceae family with the Anacardiceae and Thymelaeaceae families. Holstiinone A (1) and lophirone F (14) exhibited moderate antibacterial activity against $B$. subtilis and showed weak cytotoxicity against MCF-7, respectively.

\section{EXPERIMENTAL SECTION}

General Experimental Procedures. Optical rotations were determined using a $341 \mathrm{LC}$ OROT polarimeter $\left(589 \mathrm{~nm}, 24.0^{\circ} \mathrm{C}\right)$. ECD experiments were performed on a Jasco model J-810, Rev.1.00, spectropolarimeter. UV spectra were obtained using $\mathrm{CH}_{3} \mathrm{OH}$ as the solvent using a Shimadzu UV-1650PC UV/vis spectrophotometer. Infrared (IR) spectra were recorded on a PerkinElmer Spectrum FTIR spectrometer using liquid samples. NMR spectra were acquired on either a Bruker Avance NEO $500 \mathrm{MHz}$ (TXO cryogenic probe) or a $600 \mathrm{MHz}$ (TCI cryogenic probe) spectrometer and were processed using the MestreNova (v14.0.0) software. Chemical shifts were referenced to the carbon and residual proton signals of the deuterated solvents $\left(\mathrm{CD}_{3} \mathrm{CN} \delta_{\mathrm{H}} 1.94\right.$ and $\delta_{\mathrm{C}} 118.26$, methanol- $d_{4} \delta_{\mathrm{H}} 3.30$ and $\delta_{\mathrm{C}}$ 49.00, $\mathrm{CDCl}_{3} \delta_{\mathrm{H}} 7.26$ and $\left.\delta_{\mathrm{C}} 77.16\right)$ as internal standard. Assignments were based on ${ }^{1} \mathrm{H},{ }^{13} \mathrm{C}$, COSY, HSQC, HMBC, TOCSY, and NOESY NMR spectra. LC-ESIMS spectra were acquired on a Micromass GCTOF micromass spectrometer (Micromass, Wythenshawe, Waters, Inc. UK), recorded on a direct inlet, with $70 \mathrm{eV}$ ionization voltage. Elution was performed with a gradient mobile phase $\left(\mathrm{H}_{2} \mathrm{O}-\mathrm{CH}_{3} \mathrm{CN}\right.$, 95:05-5:95, v/v) with a run time of 4-10 min. HRESIMS spectra were obtained with a Q-TOF-LC/MS spectrometer using a $2.1 \times 30$ mm $1.7 \mu \mathrm{m} \mathrm{RPC}_{18}$ and $\mathrm{H}_{2} \mathrm{O}-\mathrm{CH}_{3} \mathrm{CN}$ gradient (5:95-95:5 in $0.2 \%$ formic acid, v/v) at Sternhagen Analys Lab AB, Gothenburg, Sweden. Thin layer chromatography (TLC) was performed on silica gel 60 $\mathrm{F}_{254}$ (Merck, Darmstadt, Germany) using precoated aluminum plates to monitor isolation processes. Various appropriate solvent systems were used to develop TLC plates, which were visualized under UV light $(254$ and $366 \mathrm{~nm})$ and spraying with an anisaldehyde reagent (prepared by mixing $3.5 \mathrm{~mL}$ of 4-anisaldehyde with $2.5 \mathrm{~mL}$ of concentrated $\mathrm{H}_{2} \mathrm{SO}_{4}, 4 \mathrm{~mL}$ of glacial $\mathrm{HOAc}$, and $90 \mathrm{~mL}$ of $\mathrm{CH}_{3} \mathrm{OH}$ ) followed by heating $\left(80-100{ }^{\circ} \mathrm{C}\right)$. Column chromatography (CC) was run on silica gel 60 (230-400 mesh). Gel filtration was performed on Sephadex LH-20 (GE Healthcare). Preparative reversed-phase HPLC was performed on an Interchim Ultra Performance Flash Purification (PF-430) system using Interchim v $5.1 \mathrm{~d} .02$ software and an $\mathrm{RP}-\mathrm{C}_{8}$ Kromasil column $(250 \mathrm{~mm} \times 25 \mathrm{~mm}$, $5 \mu \mathrm{m})$.

Plant Material. The stem bark of Ochna holstii was collected in April 2017 from the Mkwagulo and Mtakayo clans' sacred forest graveyards $\left(6^{\circ} 24^{\prime} 51.918^{\prime \prime} \mathrm{S}\right.$; $38^{\circ} 40^{\prime} 19.308^{\prime \prime} \mathrm{E}$, altitude $\left.78.80 \mathrm{~m}\right)$ in Fukayosi Village, Bagamoyo District, Pwani Region in Tanzania. The plant was identified and authenticated by F. M. Mbago, a senior taxonomist of the herbarium at the Botany Department of the University of Dar es Salaam, where a voucher specimen (FMM. 3809) was deposited.

Extraction and Isolation. The air-dried and ground stem bark $(1.7 \mathrm{~kg})$ of $\mathrm{O}$. holstii was extracted with $\mathrm{CH}_{3} \mathrm{OH}-\mathrm{CH}_{2} \mathrm{Cl}_{2}(7: 3, \mathrm{v} / \mathrm{v})$ for $72 \mathrm{~h}$ at room temperature by percolation $(3 \times 3 \mathrm{~L})$ to yield a dark brown crude extract $(86 \mathrm{~g})$ after evaporation in a rotary evaporator at $40{ }^{\circ} \mathrm{C}$. A portion of the crude extract $(63 \mathrm{~g})$ was subjected to column chromatography over silica gel $60(400 \mathrm{~g})$, and gravity elution was performed with a mixture of isohexane containing increasing amounts of EtOAc (0-100\%), followed by $5 \% \mathrm{CH}_{3} \mathrm{OH}$ in EtOAc. A total of 180 fractions were collected and combined to 20 fractions, F1-F20, on the basis of TLC analyses. F1 was subjected to a Sephadex LH-20 column $\left(\mathrm{CH}_{3} \mathrm{OH}-\mathrm{CH}_{2} \mathrm{Cl}_{2}, 8: 2\right)$ to afford white solids holstiinone B $(2,8 \mathrm{mg})$ and ouratein $\mathrm{D}^{15}(6,6 \mathrm{mg}) . \mathrm{F} 2$ was subjected to silica gel column chromatography (30\% EtOAc in isohexane) followed by purification on Sephadex $\mathrm{LH}-20\left(\mathrm{CH}_{3} \mathrm{OH}-\mathrm{CH}_{2} \mathrm{Cl}_{2}, 8: 2\right)$ to give a pale yellow solid, holstiinone $\mathrm{A}(1,6 \mathrm{mg})$. F3 and $\mathrm{F} 4$ were further combined and purified on Sephadex $\mathrm{LH}-20\left(\mathrm{CH}_{3} \mathrm{OH}-\mathrm{CH}_{2} \mathrm{Cl}_{2}, 1: 1\right)$ to afford isochamaejasmin $\mathrm{A}^{16,17}(7,6 \mathrm{mg})$. Purification of $\mathrm{F} 5$ on 
Sephadex LH-20 $\left(\mathrm{CH}_{3} \mathrm{OH}\right)$ gave a yellow solid, calodenin $\mathrm{B}^{24,25}(\mathbf{1 6}$, $50 \mathrm{mg}$ ). F6 and F7 were further combined and purified by a Sephadex LH-20 column $\left(\mathrm{CH}_{3} \mathrm{OH}-\mathrm{CH}_{2} \mathrm{Cl}_{2}, 1: 1\right)$ followed by preparative HPLC $\left(\mathrm{CH}_{3} \mathrm{OH}-\mathrm{MeCN}, 85: 15\right.$, for $40 \mathrm{~min}$, with a flow rate of 4 $\mathrm{mL} / \mathrm{min})$ to afford holstiichalcone $\mathrm{I}(3,12 \mathrm{mg})$ and flavumchalcone $(13,4 \mathrm{mg}){ }^{22}$ F8 was purified with a Sephadex LH-20 column $\left(\mathrm{CH}_{3} \mathrm{OH}\right)$ to afford a yellow solid, holstiichalcone II $(4,7 \mathrm{mg})$. F9 was subjected to silica gel column chromatography (EtOAc$\left.\mathrm{CH}_{2} \mathrm{Cl}_{2}, 1: 1\right)$ followed by repeated purification on Sephadex LH-20 $\left(\mathrm{CH}_{3} \mathrm{OH}-\mathrm{CH}_{2} \mathrm{Cl}_{2}, 8: 2\right)$ to give a yellow solid, holstiichalcone III (5, $5 \mathrm{mg}$ ). F17 and F18 were combined and purified on a Sephadex LH20 column $\left(\mathrm{CH}_{3} \mathrm{OH}-\mathrm{CH}_{2} \mathrm{Cl}_{2}, 1: 1\right)$ followed by silica gel column chromatography (EtOAc- $\mathrm{CH}_{2} \mathrm{Cl}_{2}, 1: 1$ ) to afford a yellow solid, lophirone $\mathrm{F}^{23}(14,40 \mathrm{mg})$, and dihydrocalodenin $\mathrm{B}^{3}(15,14 \mathrm{mg})$. Purification of F10 on a Sephadex LH-20 column $\left(\mathrm{CH}_{3} \mathrm{OH}\right)$ gave a white solid, campylospermone $\mathrm{A}^{19}(9,15 \mathrm{mg})$. Purification of $\mathrm{F} 11$ by preparative TLC (EtOAc- $\mathrm{CH}_{2} \mathrm{Cl}_{2}, 30: 70$ ) afforded a white solid, $7,7^{\prime \prime}$-di-O-methylchamaejasmin ${ }^{18}(8,4 \mathrm{mg}) . \mathrm{F} 12$ and $\mathrm{F} 15$ were combined and purified on a Sephadex LH-20 column $\left(\mathrm{CH}_{3} \mathrm{OH}-\right.$ $\left.\mathrm{CH}_{2} \mathrm{Cl}_{2}, 1: 1\right)$ followed by further purification by preparative HPLC $\left(\mathrm{H}_{2} \mathrm{O}-\mathrm{CH}_{3} \mathrm{CN}, 85: 15\right.$, for $60 \mathrm{~min}$ at a flow rate of $\left.4 \mathrm{~mL} / \mathrm{min}\right)$ to give liquiritigeninyl-(I-3,II-3)-naringenin ${ }^{8}(10,4 \mathrm{mg})$. F16 was purified by preparative TLC $\left(\mathrm{CH}_{3} \mathrm{OH}-\mathrm{CH}_{2} \mathrm{Cl}_{2}, 5: 95\right)$ to give a white solid, isoliquitigenin $^{20}(\mathbf{1 1}, 4 \mathrm{mg})$, and a yellow solid, terminalionone ${ }^{21}$ (12, $6 \mathrm{mg}$ ).

Holstiinone $A$ (1): pale white, amorphous solid; $[\alpha]^{24}-115(c$ $\left.0.013, \mathrm{CH}_{3} \mathrm{OH}\right)$; UV $\left(\mathrm{CH}_{3} \mathrm{OH}\right) \lambda_{\max } 330$ and $262 \mathrm{~nm}$; ECD $(c 0.05$, $\left.\mathrm{CH}_{3} \mathrm{OH}\right) \lambda_{\max }(\Delta \varepsilon) 360(6.0), 300(-45.0), 210(-50.0)$; IR $\nu_{\max }$ $3396,1604,1516,1453, \mathrm{~cm}^{-1} ;{ }^{1} \mathrm{H}$ and ${ }^{13} \mathrm{C}$ NMR see Table 1; HRESIMS $m / z 541.1487[\mathrm{M}+\mathrm{H}]^{+}\left(\right.$calcd for $\left.\mathrm{C}_{31} \mathrm{H}_{25} \mathrm{O}_{9}, 541.1499\right)$.

Holstiinone $B$ (2): white solid; $[\alpha]^{24}-92\left(c 0.013, \mathrm{CH}_{3} \mathrm{OH}\right)$; UV $\left(\mathrm{CH}_{3} \mathrm{OH}\right) \lambda_{\max } 340$ and $258 \mathrm{~nm} ; \mathrm{ECD}\left(c 0.05, \mathrm{CH}_{3} \mathrm{OH}\right) \lambda_{\max }(\Delta \varepsilon)$ 360 (6.0), 300 (-45.0), $210(-50.0)$; IR $\nu_{\max } 3320,1698,1598$, $11515, \mathrm{~cm}^{-1}$; ${ }^{1} \mathrm{H}$ and ${ }^{13} \mathrm{C}$ NMR data see Table 1; HRESIMS $\mathrm{m} / \mathrm{z}$ $569.1741[\mathrm{M}+\mathrm{H}]^{+}$(calcd for $\left.\mathrm{C}_{33} \mathrm{H}_{29} \mathrm{O}_{9}, 569.1812\right)$.

Holstiichalcone I (3): yellow, amorphous solid; $[\alpha]_{\mathrm{D}}^{24}-176.9(c$ $0.23 \mathrm{CH}_{3} \mathrm{OH}$ ); UV $\mathrm{CH}_{3} \mathrm{OH} \lambda_{\max } 340$ and $264 \mathrm{~nm}$; ECD (c 0.013 , $\left.\mathrm{CH}_{3} \mathrm{OH}\right) \lambda_{\text {max }}(\Delta \varepsilon) 224(-47), 284(-33), 361(+4) \mathrm{nm}$; IR $v_{\text {max }}$ 3305, 1700, 1598, 1513, $\mathrm{cm}^{-1} ;{ }^{1} \mathrm{H}$ and ${ }^{13} \mathrm{C}$ NMR see Table 2; HRESIMS $m / z$ 567.1634 $[\mathrm{M}+\mathrm{Na}]^{+}\left(\right.$calcd for $\left.\mathrm{C}_{31} \mathrm{H}_{28} \mathrm{O}_{9} 567.1631\right)$.

Holstiichalcone II (4): yellow solid; $[\alpha]^{24}-21\left(c 0.013, \mathrm{CH}_{3} \mathrm{OH}\right)$; UV CH$~_{3} \mathrm{OH} \lambda_{\max } 368,340$, and $284 \mathrm{~nm}$; ECD $\left(c 0.05, \mathrm{CH}_{3} \mathrm{OH}\right) \lambda_{\max }$ $(\Delta \varepsilon) 436(-7), 373(-6) 320(-6.0), 293(4.0), 274(-4), 253$ (5.0), 230 (-18), 204 (20); IR $\nu_{\max } 3382,1604,1554,1515,1444$, $1363 \mathrm{~cm}^{-1}$; ${ }^{1} \mathrm{H}$ and ${ }^{13} \mathrm{C}$ NMR see Table 3; HRESIMS $\mathrm{m} / z 511.1478$ $[\mathrm{M}+\mathrm{H}]^{+}$(calcd for $\left.\mathrm{C}_{30} \mathrm{H}_{23} \mathrm{O}_{8}, 511.1393\right)$.

Holstiichalcone III (5): yellow solid; $\mathrm{UV} \mathrm{CH}_{3} \mathrm{OH} \lambda_{\max } 336,320$, and $264 \mathrm{~nm}$; IR $\nu_{\max } 3326,1709,1506,1515 \mathrm{~cm}^{-1} ;{ }^{1} \mathrm{H}$ and ${ }^{13} \mathrm{C}$ NMR data see Table 4; HRESIMS $\mathrm{m} / z 513.1575[\mathrm{M}+\mathrm{H}]^{+}$(calcd for $\mathrm{C}_{30} \mathrm{H}_{25} \mathrm{O}_{8}$, 513.1549).

Antibacterial Assays. The antibacterial activity of the crude extract and the isolated compounds was evaluated against two bacterial strains, B. subtilis (Gram-positive) and E. coli (Gramnegative). Each test sample was first dissolved at $10 \mathrm{mg} / \mathrm{mL}$ in $100 \%$ DMSO and stored at $-20{ }^{\circ} \mathrm{C}$. The bacterial strains were cultured by standard procedures as previously reported. ${ }^{52}$ Briefly, a culture of bacterial cells was grown to optical density (OD) $600_{\mathrm{nm}}=0.5$, then diluted $(10 \times)$ with prewarmed medium. The substances to be tested were added to culture medium for a final concentration of $30 \mu \mathrm{g} / \mathrm{mL}$, each at $100 \mu \mathrm{L}$ in a 96-well microtiter plate, then incubated for $24 \mathrm{~h}$ at $37{ }^{\circ} \mathrm{C}$ without agitation. The cell viability was measured by a resazurin-based assay as previously described. ${ }^{53}$ Subsequently, $12 \mu \mathrm{L}$ of AlamarBlue solution was added to each well and incubated at 37 ${ }^{\circ} \mathrm{C}$ continuously for $1 \mathrm{~h}$. The fluorescence was measured using a POLARstar Omega microplate reader from BMG Labtech with the excitation filter set to $544 \mathrm{~nm}$ and emission filter to $590 \mathrm{~nm}$. Ampicillin, a standard antibiotic, was used as a positive control, while DMSO, the solvent used to dissolve the test substances, was used as a negative control. In order to prevent fluorescence bleed-through from the resorufin dye, the assays performed in 384-well plates were set up with an empty well in-between. Certain substances were checked for possible fluorescent or fluorescence-quenching properties by adding the substance after the culture was incubated with the resazurin dye only. The results were then compared to the fluorescence values obtained initially without the addition of the compound/extract. All experiments were performed in triplicate. The test compound/ extracts that caused a reduction of fluorescence by at least $50 \%$ relative to the solvent control in any of the species were followed up by additional tests to accurately determine antibacterial activity.

Cytotoxicity Assay. MCF-7 cells were cultured in Dulbecco's modified Eagle's medium supplemented with $10 \%$ fetal calf serum and kept in exponential growth as described previously. ${ }^{54}$ Briefly, cells were seeded in 96-well plates at a density of $5 \times 10^{3}$ per well allowing continued exponential growth overnight. ${ }^{55}$ Stock solutions of the test sample dissolved in DMSO were added for a final concentration of $0.3 \% \mathrm{v} / \mathrm{v}$ of the solvent in the culture medium. Cell viability was assayed using PrestoBlue cell viability reagent (ThermoFisher) according to the manufacturer's instructions following $24 \mathrm{~h}$ of incubation in the presence of the compounds/extracts. A POLARstar Omega plate reader (BMG Lab Tech) was used to measure resorufin fluorescence at $544 \mathrm{~nm}$ excitation/590 nm emission. The cells treated only with DMSO were used as a negative control. ${ }^{55}$ Three independent replicate experiments were used to calculate the $\mathrm{EC}_{50}$ value for each compound/extract using 2 -fold serial dilution.

\section{ASSOCIATED CONTENT}

\section{Supporting Information}

The original NMR spectra for all compounds, along with the corresponding NMReDATA ${ }^{56}$ for the new compounds 1-5, are available free of charge on Zenodo as DOI: 10.5281/ zenodo.4030263. The Supporting Information is available free of charge at https://pubs.acs.org/doi/10.1021/acs.jnatprod.0c01017.

NMR and MS data for the isolated compounds (1-16) (PDF)

\section{AUTHOR INFORMATION}

\section{Corresponding Authors}

Mate Erdelyi - Department of Chemistry - BMC, Uppsala University, SE-751 23 Uppsala, Sweden; —o orcid.org/00000003-0359-5970; Phone: +46-72-9999166;

Email: mate.erdelyi@kemi.uu.se

Stephen S. Nyandoro - Chemistry Department, College of Natural and Applied Sciences, University of Dar es Salaam, Dar es Salaam, Tanzania; Phone: +255-754-206560; Email: nyandoro@udsm.ac.tz

\section{Authors}

Thobias M. Kalenga - Chemistry Department, College of Natural and Applied Sciences, University of Dar es Salaam, Dar es Salaam, Tanzania

Monica M. Ndoile - Chemistry Department, College of Natural and Applied Sciences, University of Dar es Salaam, Dar es Salaam, Tanzania

Yoseph Atilaw - Department of Chemistry - BMC, Uppsala University, SE-751 23 Uppsala, Sweden

Pieter J. Gilissen - Institute for Molecules and Materials, Radboud University, 6525 AJ Nijmegen, The Netherlands

Joan J. E. Munissi - Chemistry Department, College of Natural and Applied Sciences, University of Dar es Salaam, Dar es Salaam, Tanzania

Anastasia Rudenko - Department of Chemistry and Molecular Biology, University of Gothenburg, SE-405 30 Gothenburg, Sweden; Centre for Antibiotic Resistance 
Research (CARe) at the University of Gothenburg, 40530 Gothenburg, Sweden

Catarina Bourgard - Department of Chemistry and Molecular Biology, University of Gothenburg, SE-405 30 Gothenburg, Sweden; Centre for Antibiotic Resistance Research (CARe) at the University of Gothenburg, 40530 Gothenburg, Sweden

Per Sunnerhagen - Department of Chemistry and Molecular Biology, University of Gothenburg, SE-405 30 Gothenburg, Sweden; Centre for Antibiotic Resistance Research (CARe) at the University of Gothenburg, 40530 Gothenburg, Sweden; (1) orcid.org/0000-0002-0967-8729

Complete contact information is available at: https://pubs.acs.org/10.1021/acs.jnatprod.0c01017

\section{Notes}

The authors declare no competing financial interest.

\section{ACKNOWLEDGMENTS}

Financial support from the Swedish Research Council (201903715), the Centre for Antibiotic Resistance Research (CARe) at the University of Gothenburg, and the Ministry of Education, Science and Technology, Republic of Tanzania, is highly appreciated. This study made use of the NMR Uppsala infrastructure, which is funded by the Department of Chemistry - BMC and the Disciplinary Domain of Medicine and Pharmacy. Mr. Mbago is acknowledged for locating and identifying the plant species.

\section{REFERENCES}

(1) Bandi, A. K. R.; Lee, D. U.; Tih, R. G.; Gunasekar, D.; Bodo, B. Chem. Biodiversity 2012, 9, 251-271.

(2) Makhafola, T. J.; Samuel, B. B.; Elgorashi, E. E.; Eloff, J. N. Nat. Prod. Commun. 2012, 7, 1601-1604.

(3) Tang, S.; Bremner, P.; Kortenkamp, A.; Schlage, C.; Gray, A. I.; Gibbons, S.; Heinrich, M. Planta Med. 2003, 69, 247-253.

(4) Awadh, M.; Tarus, P.; Onani, M.; Machocho, A.; Hassanali, A. Nat. Prod. Chem. Res. 2014, 2, 1000149.

(5) Adem, F. A.; Mbaveng, A. T.; Kuete, V.; Heydenreich, M.; Ndakala, A.; Irungu, B.; Yenesew, A.; Efferth, T. Phytomedicine 2019, $58,152853$.

(6) Ichino, C.; Kiyohara, H.; Soonthornchareonnon, N.; Chuakul, W.; Ishiyama, A.; Sekiguchi, H.; Namatame, M.; Otoguro, K.; Omura, S.; Yamada, H. Planta Med. 2006, 72, 611-614.

(7) Mdee, L. K.; Yeboah, S. O.; Abegaz, B. M. J. Nat. Prod. 2003, 66, 599-604.

(8) Dhooghe, L.; Maregesi, S.; Mincheva, I.; Ferreira, D.; Marais, J. P.; Lemiere, F.; Matheeussen, A.; Cos, P.; Maes, L.; Vlietinck, A.; Apers, S.; Pieters, L. Phytochemistry 2010, 71, 785-791.

(9) Verdcourt, B.; Beentje, H. Flora of Tropical East Africa: Selaginellaceae; Royal Botanic Gardens Kew: Tanzania, 2005; Vol. 225, pp 7-39.

(10) Gebretsadik, Z. M.; Telila, A. N. World. J. Biol. Biol. Sci. 2013, 1, $1-9$.

(11) Geldenhuys, C.; Pieterse, F. S. A. Forest. J. 1993, 164, 9-19.

(12) Sigidi, M. T.; Traoré, A. N.; Boukandou, M. M.; Tshisikhawe, M. P.; Ntuli, S. S.; Potgieter, N. Indian. J. Tradit. Know. 2017, 16, 545-552.

(13) Parker, M. E.; Chabot, S.; Ward, B. J.; Johns, T. J. Ethnopharmacol. 2007, 114, 146-152.

(14) Tsegaye, D. W. Phytochemical investigation of selected millettia (leguminosae) and ochna (ochnaceae) species for anticancer activities $\mathrm{PhD}$, Thesis, University of Nairobi, 2015.

(15) Rocha, M. P.; Campana, P. R.; Paídua, R. M.; Souza Filho, J. D.; Ferreira, D.; Braga, F. C. J. Nat. Prod. 2020, 83, 1891-1898.

(16) Niwa, M.; Chen, X.-F.; Liu, G.-Q.; Tatematsu, H.; Hirata, Y. Chem. Lett. 1984, 13, 1587-1590.
(17) Niwa, M.; Otsuji, S.; Tatematsu, H.; Liu, G.-Q.; Chen, X.-F.; Hirata, Y. Chem. Pharm. Bull. 1986, 34, 3249-3251.

(18) Chukwujekwu, J. C.; De Kock, C. A.; Smith, P. J.; Van Heerden, F. R.; Van Staden, J. Planta Med. 2012, 78, 1857-1860.

(19) Manga, S. E.; Tih, A.; Ghogomu, R.; Blond, A.; Bodo, B. Biochem. Syst. Ecol. 2009, 37, 402-404.

(20) Kong, L.; Zhang, Y.; Pan, X.; Tan, R.; Cheng, C. Cell. Mol. Life Sci. 2000, 57, 500-505.

(21) Negishi, H.; Maoka, T.; Njelekela, M.; Yasui, N.; Juman, S.; Mtabaji, J.; Miki, T.; Nara, Y.; Yamori, Y.; Ikeda, K. J. Asian Nat. Prod. Res. 2011, 13, 281-283.

(22) Ndongo, J. T.; Shaaban, M.; Mbing, J. N.; Bikobo, D. N.; de Théodore Atchadé, A.; Pegnyemb, D. E.; Laatsch, H. Phytochemistry 2010, 71, 1872-1878.

(23) Tih, R. G.; Sondengam, B.; Martin, M.; Bodo, B. Phytochemistry 1990, 29, 2289-2293.

(24) Messanga, B.; Tih, R. G.; Sondengam, B.-L.; Martin, M.-T.; Bodo, B. Phytochemistry 1994, 35, 791-794.

(25) Drewes, S. E.; Hudson, N. A.; Bates, R. B.; Linz, G. S. J. Chem. Soc., Perkin Trans. 1 1987, 2809-2813.

(26) Kumar, S. Cosm. Rays. 2006, 10, 4.

(27) Ndoile, M. M.; Van Heerden, F. R. Int. Res. J. Pure Appl. Chem. 2018, 16, 1-9.

(28) Yang, G.; Liao, Z.; Xu, Z.; Zhang, H.; Chen, D. Chem. Pharm. Bull. 2005, 53, 776-779.

(29) Maeda, G.; van der Wal, J.; Gupta, A. K.; Munissi, J. J.; Orthaber, A.; Sunnerhagen, P.; Nyandoro, S. S.; Erdeílyi, M. J. Nat. Prod. 2020, 83, 210-215.

(30) Schroder, G.; Wehinger, E.; Lukacin, R.; Wellmann, F.; Seefelder, W.; Schwab, W.; Schroder, J. Phytochemistry 2004, 65, $1085-1094$

(31) Anuradha, V.; Srinivas, P. V.; Rao, R. R.; Manjulatha, K.; Purohit, M. G.; Rao, J. M. Bioorg. Med. Chem. 2006, 14, 6820-6826.

(32) Tang, S.; Bremner, P.; Kortenkamp, A.; Schlage, C.; Gray, A. I.; Gibbons, S.; Heinrich, M. Planta Med. 2003, 69, 247-253.

(33) Mbabi Nyemeck, N., 2nd; Serge Ngono Bikobo, D.; Abouem, A. Z. A.; Schafer, E. M.; Bochet, C.; Emmanuel Pegnyemb, D.; Koert, U. Nat. Prod. Res. 2017, 31, 2875-2884.

(34) Tih, A.; Tih, G.; Sondengam, B.; Martin, M.; Bodo, B. Tetrahedron Lett. 1988, 29, 5797-5800.

(35) Murakami, A.; Tanaka, S.; Ohigashi, H.; Hirota, M.; Irie, R.; Takeda, N.; Tatematsu, A.; Koshimizu, K. Phytochemistry 1992, 31, 2689-2693.

(36) Covington, C. L.; Junior, F. M.; Silva, J. H.; Kuster, R. M.; de Amorim, M. B.; Polavarapu, P. L. J. Nat. Prod. 2016, 79, 2530-2537.

(37) Jang, H.; Lee, J. W.; Jin, Q.; Kim, S. Y.; Lee, D.; Hong, J. T.; Kim, Y.; Lee, M. K.; Hwang, B. Y. Helv. Chim. Acta 2015, 98, 14191425.

(38) Lin, G.-Q.; Zhong, M. Tetrahedron Lett. 1997, 38, 1087-1090.

(39) Feng, B.; Pei, Y.; Hua, H.; Wang, T.; Zhang, Y. Pharm. Biol. 2003, 41, 59-61.

(40) Harada, N.; Ono, H.; Uda, H.; Parveen, M.; Khan, N. U. D.; Achari, B.; Dutta, P. K. J. Am. Chem. Soc. 1992, 114, 7687-7692.

(41) Jang, H.; Lee, J. W.; Jin, Q. H.; Kim, S. Y.; Lee, D.; Hong, J. T.; Kim, Y.; Lee, M. K.; Hwang, B. Y. Helv. Chim. Acta 2015, 98, 14191425.

(42) Zhang, F. J.; Lin, G. Q.; Huang, Q. C. J. Org. Chem. 1995, 60, 6427-6430

(43) Sisa, M.; Bonnet, S. L.; Ferreira, D.; Van der Westhuizen, J. H. Molecules 2010, 15, 5196-5245.

(44) de Carvalho, M. G.; Alves, C. C. F.; da Silva, K. G.; Eberlin, M. N.; Werle, A. A. J. Braz. Chem. Soc. 2004, 15, 146-149.

(45) Cardoso, M. P.; Lima, L. S.; David, J. P.; Moreira, B. O.; Santos, E. O.; David, J. M.; Alves, C. Q. J. Braz. Chem. Soc. 2015, 26, 15271531.

(46) Liu, G.; Tatematsu, H.; Kurokawa, M.; Niwa, M.; Hirata, Y. Chem. Pharm. Bull. 1984, 32, 362-365. 
(47) Wang, Z.-X.; Cheng, M.-X.; Zhang, X.-Z.; Hong, Z.-L.; Gao, M.-Z.; Kan, X.-X.; Li, Q.; Wang, Y.-J.; Zhu, X.-X.; Xiao, H.-B. Fitoterapia 2014, 99, 334-340.

(48) Niwa, M.; Tatematsu, H.; Liu, G.-Q.; Hirata, Y. Chem. Lett. 1984, 13, 539-542.

(49) Bermudez, J.; Rodriguez, M.; Hasegawa, M.; Gonzalez-Mujica, F.; Duque, S.; Ito, Y. Nat. Prod. Commun. 2012, 7, 973-976.

(50) Dhooghe, L.; Maregesi, S.; Mincheva, I.; Ferreira, D.; Marais, J. P.; Lemière, F.; Matheeussen, A.; Cos, P.; Maes, L.; Vlietinck, A.; Apers, S.; Luc, P. Phytochemistry 2010, 71, 785-791.

(51) Yang, G.; Chen, D. Chem. Biodiversity 2008, 5, 1419-1424.

(52) Chen, S.; Liu, Y.; Liu, Z.; Cai, R.; Lu, Y.; Huang, X.; She, Z. RSC Adv. 2016, 6, 26412-26420.

(53) Sarker, S. D.; Nahar, L.; Kumarasamy, Y. Methods 2007, 42, 321-324.

(54) Koudokpon, H.; Armstrong, N.; Dougnon, T.; Fah, L.; Hounsa, E.; Bankolé, H.; Loko, F.; Chabrière, E.; Rolain, J. Biomed. Res. Int. 2018, 2018.

(55) Chen, S.; Chen, D.; Cai, R.; Cui, H.; Long, Y.; Lu, Y.; Li, C.; She, Z. J. Nat. Prod. 2016, 79, 2397-2402.

(56) Pupier, M.; Nuzillard, J. M.; Wist, J.; Schlorer, N. E.; Kuhn, S.; Erdelyi, M.; Steinbeck, C.; Williams, A. J.; Butts, C.; Claridge, T. D. W.; Mikhova, B.; Robien, W.; Dashti, H.; Eghbalnia, H. R.; Fares, C.; Adam, C.; Kessler, P.; Moriaud, F.; Elyashberg, M.; Argyropoulos, D.; Perez, M.; Giraudeau, P.; Gil, R. R.; Trevorrow, P.; Jeannerat, D. Magn. Reson. Chem. 2018, 56, 703-715. 